\title{
TURBULENT MOLECULAR GAS AND STAR FORMATION IN THE SHOCKED INTERGALACTIC MEDIUM OF STEPHAN'S QUINTET
}

\author{
P. Guillard ${ }^{1,2}$, F. Boulanger ${ }^{2}$, G. Pineau des Forêts ${ }^{2,3}$, E. Falgarone ${ }^{3}$, A. Gusdorf ${ }^{3}$, M. E. Cluver ${ }^{1}$, \\ P. N. ApPleton ${ }^{6}$, U. Lisenfeld ${ }^{1,7,8}$, P.-A. Duc ${ }^{9}$, P. M. OGLE ${ }^{1}$, ANd C. K. Xu ${ }^{10}$ \\ ${ }^{1}$ Spitzer Science Center (SSC), California Institute of Technology, MC 220-6, Pasadena, CA 91125, USA \\ ${ }^{2}$ Institut d'Astrophysique Spatiale (IAS), UMR 8617, CNRS, Université Paris-Sud 11, Bâtiment 121, 91405 Orsay Cedex, France \\ ${ }^{3}$ ENS, LERMA, UMR 8112, CNRS, Observatoire de Paris, 24 rue Lhomond 75005 Paris, France \\ ${ }^{4}$ Max Planck Institut für Radioastronomie, Auf dem Hügel 69, 53121 Bonn, Germany \\ ${ }^{5}$ Laboratoire Univers et Théories (LUTH), UMR 8102 CNRS, Observatoire de Paris, Université Paris Diderot, 5 Place Jules Janssen, 92190 Meudon, France \\ ${ }^{6}$ NASA Herschel Science Center (NHSC), California Institute of Technology, Mail code 100-22, Pasadena, CA 91125, USA \\ ${ }^{7}$ Departamento de Física Teórica y del Cosmos, Universidad de Granada, Granada, Spain \\ ${ }^{8}$ Instituto de Astrofísica de Andalucía, CSIC, Apdo. 3004, 18080 Granada, Spain \\ ${ }^{9}$ AIM, Unité Mixte de Recherche CEA-CNRS, Université Paris VII, UMR 7158, France \\ ${ }^{10}$ Infrared Processing and Analysis Center (IPAC), JPL, Pasadena, CA 91109, USA \\ Received 2011 May 16; accepted 2012 February 13; published 2012 April 4
}

\begin{abstract}
The Stephan's Quintet (hereafter SQ) is a template source to study the impact of galaxies interaction on the physical state and energetics of their gas. We report on IRAM single-dish CO observations of the SQ compact group of galaxies. These observations follow up the Spitzer discovery of bright mid-IR $\mathrm{H}_{2}$ rotational line emission $\left(L\left(\mathrm{H}_{2}\right) \approx 10^{35} \mathrm{~W}\right)$ from warm $\left(10^{2-3} \mathrm{~K}\right)$ molecular gas, associated with a $30 \mathrm{kpc}$ long shock between a galaxy, NGC 7318b, and NGC 7319's tidal arm. We detect CO(1-0), (2-1) and (3-2) line emission in the inter-galactic medium (IGM) with complex profiles, spanning a velocity range of $\approx 1000 \mathrm{~km} \mathrm{~s}^{-1}$. The spectra exhibit the pre-shock recession velocities of the two colliding gas systems $\left(5700\right.$ and $\left.6700 \mathrm{~km} \mathrm{~s}^{-1}\right)$, but also intermediate velocities. This shows that much of the molecular gas has formed out of diffuse gas accelerated by the galaxy-tidal arm collision. CO emission is also detected in a bridge feature that connects the shock to the Seyfert member of the group, NGC 7319, and in the northern star forming region, SQ-A, where a new velocity component is identified at $6900 \mathrm{~km} \mathrm{~s}^{-1}$, in addition to the two velocity components already known. Assuming a Galactic $\mathrm{CO}(1-0)$ emission to $\mathrm{H}_{2}$ mass $^{2}$ conversion factor, a total $\mathrm{H}_{2}$ mass of $\approx 5 \times 10^{9} M_{\odot}$ is detected in the shock. The ratio between the warm $\mathrm{H}_{2}$ mass derived from Spitzer spectroscopy, and the $\mathrm{H}_{2}$ mass derived from CO fluxes is $\approx 0.3$ in the IGM of $\mathrm{SQ}$, which is 10-100 times higher than in star-forming galaxies. The molecular gas carries a large fraction of the gas kinetic energy involved in the collision, meaning that this energy has not been thermalized yet. The kinetic energy of the $\mathrm{H}_{2}$ gas derived from $\mathrm{CO}$ observations is comparable to that of the warm $\mathrm{H}_{2}$ gas from Spitzer spectroscopy, and a factor $\approx 5$ greater than the thermal energy of the hot plasma heated by the collision. In the shock and bridge regions, the ratio of the PAH-to-CO surface luminosities, commonly used to measure the star formation efficiency of the $\mathrm{H}_{2}$ gas, is lower (up to a factor 75) than the observed values in star-forming galaxies. We suggest that turbulence fed by the galaxy-tidal arm collision maintains a high heating rate within the $\mathrm{H}_{2}$ gas. This interpretation implies that the velocity dispersion on the scale of giant molecular clouds in SQ is one order of magnitude larger than the Galactic value. The high amplitude of turbulence may explain why this gas is not forming stars efficiently.
\end{abstract}

Key words: galaxies: clusters: individual: Stephan's Quintet - galaxies: interactions - galaxies: ISM - intergalactic medium

Online-only material: color figures

\section{INTRODUCTION}

Galaxy interactions represent an important agent of galaxy evolution, involving the injection of huge amounts of kinetic energy in the interstellar medium. Galaxy collisions or interactions of galaxies with the intergalactic medium (IGM) may trigger bursts of star formation detected in the infrared (IR, e.g., Joseph \& Wright 1985; Harwit et al. 1987; Kim et al. 1995; $\mathrm{Xu}$ et al. 1999). Star formation proceeds where and when the kinetic energy of the gas is being radiated, allowing gas to cool and condense. To make headway in our understanding of galaxy evolution and, in particular, the star formation history of the universe, it is crucial to understand how this kinetic energy is dissipated, and how this dissipation affects the passage from molecular gas to stars.

Stephan's Quintet (Hickson Compact Group HCG 92, hereafter SQ) is an ideal laboratory to study these physical processes. SQ is a group of five interacting galaxies, with a complex dynamical history (for a review see Xu 2006 and references therein). The most striking feature of the group is that a galaxy-scale $\left(\approx 15 \times 35 \mathrm{kpc}^{2}\right)$ shock is created by an intruding galaxy, NGC 7318b, which is colliding into the intragroup gas at a relative velocity of $\approx 1000 \mathrm{~km} \mathrm{~s}^{-1}$ (see the background image in Figure 1). This IGM material is likely to be gas that has been tidally stripped from NGC 7319 by a past interaction with another galaxy, NGC 7320c (see Moles et al. 1997; Sulentic et al. 2001 for a plausible dynamical scenario). These ideas have been further explored recently with numerical simulations. Both $N$-body (Renaud et al. 2010) and hydrodynamical models (Hwang et al. 2012) of the quintet suggest a sequence of serial collisions between various group members culminating in the current high-speed shock heating of gas as NGC 7318b strikes the pre-existing tidal debris from NGC 7319. 


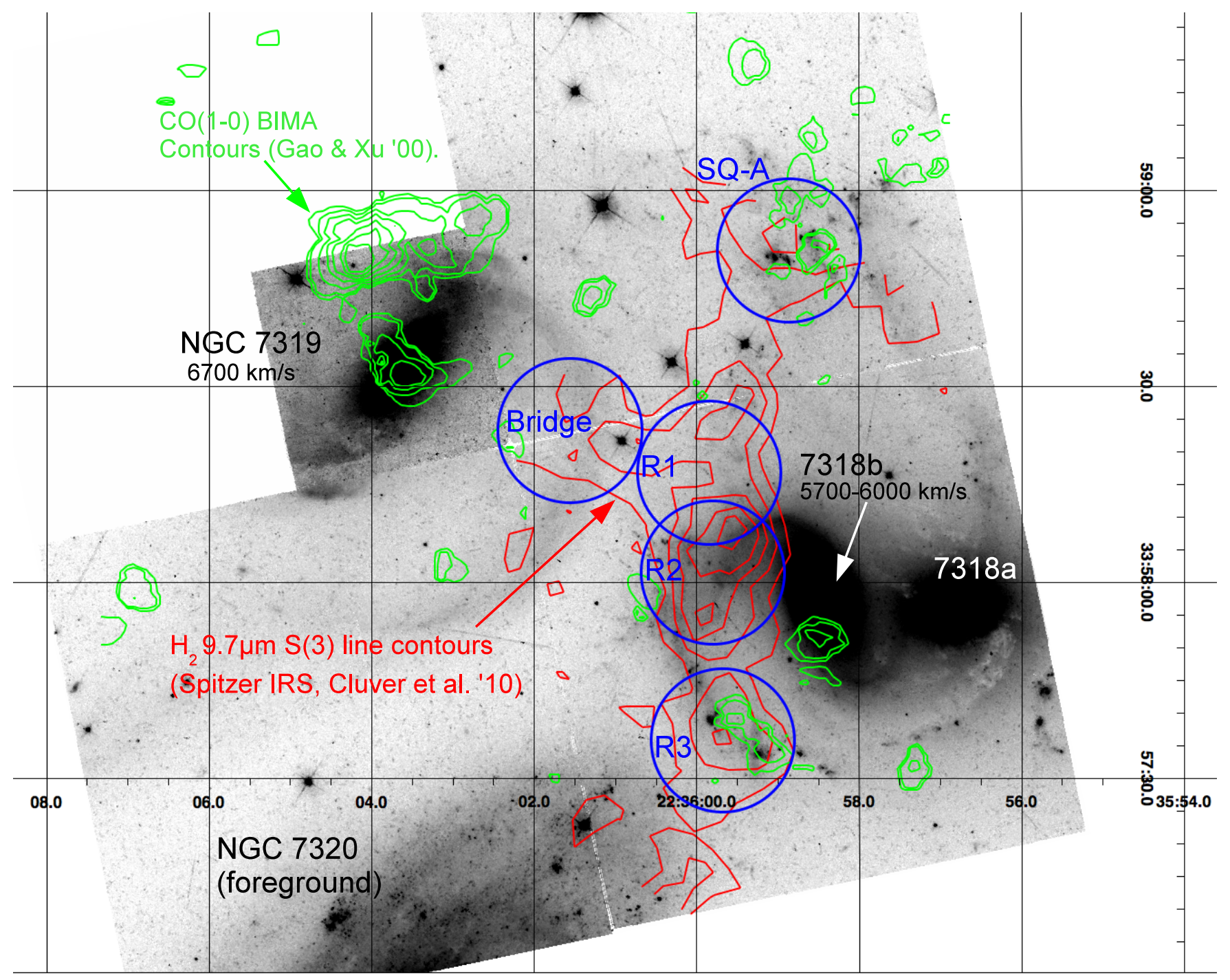

Figure 1. $\mathrm{CO}$ and $\mathrm{H}_{2}$ observations in the Stephan's Quintet group. The gray-scale background image is a $H S T$ WFC2 $V$-band image. The blue circles (22 arcsec in diameter) show the IRAM $30 \mathrm{~m} \mathrm{CO}(1-0)$ half-power beams where the observations presented in this paper have been taken. The corresponding spectra are shown on Figure 2. CO emission is detected at all these positions. The intruder galaxy, NGC 7318b, is colliding with intra-group gas (tidal debris from NGC 7319) at a relative velocity of $\sim 900 \mathrm{~km} \mathrm{~s}^{-1}$. The $\mathrm{CO}(1-0)$ green contours from BIMA observations by Gao \& Xu (2000) correspond to levels of 2.4, 3.0, 4.0, 5.0, 6.0, 7.0, and 8 $\sigma$ $\left(1 \sigma=0.7 \mathrm{Jy} \mathrm{km} \mathrm{s}^{-1}\right)$. No CO was detected in the ridge because of limited sensitivity and bandwidth. Also note that the two $\sim 3 \sigma-4 \sigma \mathrm{CO}$ features in the south were just outside (but near the edge) of the BIMA primary beam. The red contours are the $\mathrm{H}_{2} 9.7 \mu \mathrm{m} \mathrm{S}(3)$ line contours from Spitzer IRS spectral mapping by Cluver et al. (2010), which clearly shows the kpc-scale shock produced by the galaxy collision. The $\mathrm{H}_{2}$ contours are $[1.0,1.6,2.3,2.9,3.6,4.2,4.9] \times 10^{-9} \mathrm{~W} \mathrm{~m}^{-2} \mathrm{sr}^{-1}$.

(A color version of this figure is available in the online journal.)

A ridge of X-ray (Trinchieri et al. 2003, 2005) and strong radio synchrotron (e.g., Sulentic et al. 2001; Williams et al. 2002) emission from the hot $\left(5-7 \times 10^{6} \mathrm{~K}\right)$ post-shock plasma is associated with the group-wide shock. Excitation diagnostics from optical (Xu et al. 2003) and mid-IR (Cluver et al. 2010) emission lines also confirm the presence of shocked gas in the IGM. Spitzer mid-IR observations reveal that the mid-IR spectrum in the shock structure is dominated by the rotational line emission of molecular hydrogen, $\mathrm{H}_{2}$ (Appleton et al. 2006; Cluver et al. 2010). This denotes the presence of large amounts $\left(\approx 10^{9} M_{\odot}\right)$ of warm $\left(10^{2}-10^{3} \mathrm{~K}\right) \mathrm{H}_{2}$, cospatial with the X-ray emitting plasma of the group-wide shock. The $\mathrm{H}_{2}$ emission is extended (see the $\mathrm{H}_{2}$ red contours on Figure 1) along the ridge feature, toward the Seyfert galaxy NGC 7319 (in the so-called bridge), as well as in the northern intergalactic starburst SQ-A (Xu et al. 1999). Over the main shock area observed by Spitzer, the $\mathrm{H}_{2}$ luminosity $\left(L\left(\mathrm{H}_{2}\right) \approx 2.6 \times 10^{8} L_{\odot}\right.$, summed over the
$0-0 S(0)$ to $S(5)$ lines) is larger by a factor of three than that of the X-ray emission (integrated over $0.001-10 \mathrm{keV}$ ) from the same region. With the high resolution $(\mathcal{R}=600)$ of Spitzer Infrared Spectrograph (IRS), the $17 \mu \mathrm{m} \mathrm{H}_{2} \mathrm{~S}(1)$ line is resolved with an FWHM of $870 \mathrm{~km} \mathrm{~s}^{-1}$ (Appleton et al. 2006).

Guillard et al. (2009) proposed a model for the formation of molecular gas in the ridge, considering the collision of multiphase gas. In their scenario, $\mathrm{H}_{2}$ is formed from the cooling of shocked atomic gas. The shock velocity depends on the pre-shock gas density: it is high $\left(\approx 600-800 \mathrm{~km} \mathrm{~s}^{-1}\right)$ in the tenuous $\left(n_{\mathrm{H}}<0.02 \mathrm{~cm}^{-3}\right)$ gas, producing the X-ray emission in the ridge, and much lower in the dense gas. Gas at preshock densities $n_{\mathrm{H}}>0.2 \mathrm{~cm}^{-3}$ experience shock velocities small enough $\left(V_{\mathrm{s}}<200 \mathrm{~km} \mathrm{~s}^{-1}\right)$ to retain most of its dust, cool, and become molecular within a few million years. We proposed that the $\mathrm{H}_{2}$ emission is associated with the dissipation of the kinetic energy of the galaxy collision at sub-parsec scales, 
through supersonic turbulence in the molecular gas. The midIR spectral energy distribution (SED) of the $\mathrm{H}_{2}$ emission can be explained if the dense $\left(n_{\mathrm{H}}>10^{3} \mathrm{~cm}^{-3}\right)$ molecular gas is continuously processed by low-velocity (5-20 $\mathrm{km} \mathrm{s}^{-1}$ ) MHD shocks.

Since the mid-IR rotational $\mathrm{H}_{2}$ line emission only samples warm molecular gas with temperatures $\approx 10^{2}-10^{3} \mathrm{~K}$, our census of the molecular gas in the SQ shock is still incomplete. The discovery of powerful mid-IR $\mathrm{H}_{2}$ line emission in SQ led us to search for $\mathrm{CO}$ line emission associated with the warm $\mathrm{H}_{2}$ in the shock. In galaxies, the warm $\mathrm{H}_{2}$ is a small fraction $(0.4 \%-4 \%)$ of the total mass of molecular gas (Roussel et al. 2007), which is too cold $(<100 \mathrm{~K})$ to be seen in mid-IR $\mathrm{H}_{2}$ emission, and usually traced with $\mathrm{CO}$. This paper reports on the detection of CO line emission in the IGM of the SQ group and investigates the physical and dynamical state of the molecular gas in SQ, as well as the connection between shock and star formation in the group.

After reviewing the past $\mathrm{CO}$ observations of SQ (Section 2), we present technical details about our observations with the IRAM $30 \mathrm{~m}$ and the Atacama Pathfinder EXperiment (APEX) telescopes (Section 3). Then we describe the kinematics (Section 4), the distribution and mass (Section 5), the excitation and energetics (Section 6) of the molecular gas in the IGM of the group. Section 7 discusses the origin of the $\mathrm{CO}$ gas, its kinematics, and the efficiency of star formation in the group. Conclusions and final remarks are given in Section 8.

Throughout this paper we assume the distance to the SQ group to be $94 \mathrm{Mpc}$ (with a Hubble constant of $70 \mathrm{~km} \mathrm{~s}^{-1} \mathrm{Mpc}^{-2}$ ) and a systemic velocity for the group as a whole of $6600 \mathrm{~km} \mathrm{~s}^{-1}$. At this distance, $10 \operatorname{arcsec}=4.56 \mathrm{kpc}$.

\section{PREVIOUS CO OBSERVATIONS IN THE SQ GROUP}

Before the discovery of warm $\mathrm{H}_{2}$ in the IGM of the SQ group, previous observations were mostly concentrated on the Seyfert galaxy NGC 7319, on the southern tidal features, or on the northern starburst region (SQ-A). The first millimeter CO observations in SQ detected cold molecular gas in NGC 7319 (Yun et al. 1997; Verdes-Montenegro et al. 1998; Leon et al. 1998), which contains $4.8 \times 10^{9} M_{\odot}$ (Smith \& Struck 2001). Using the BIMA ${ }^{11}$ interferometer, Gao \& Xu (2000) and Petitpas \& Taylor (2005) showed that $3 / 4$ of the CO gas in NGC 7319 is lying outside its nucleus, mostly in an extended northern region (see the green contours on Figure 1).

Molecular gas outside galactic disks, associated with the IGM starburst SQ-A, was first detected by Gao \& Xu (2000) using BIMA. Then, single-dish observations by Smith \& Struck (2001) and Lisenfeld et al. (2002) confirmed this result. Two velocity components were detected, one centered at $\sim 6000 \mathrm{~km} \mathrm{~s}^{-1}$ and the other at $\sim 6700 \mathrm{~km} \mathrm{~s}^{-1}$. These velocities match the redshifts of the two Hi gas systems found in the same region (Sulentic et al. 2001; Williams et al. 2002), which correspond to the intruder galaxy, NGC 7318b, and NGC 7319's tidal tail in the IGM, respectively. Lisenfeld et al. (2002) found that there is more molecular gas $\left(3.1 \times 10^{9} M_{\odot}\right)$ in SQ-A than $\mathrm{H}_{\mathrm{I}}$ gas $\left(1.6 \times 10^{9} M_{\odot}\right)$.

Past observations with the BIMA interferometer did not lead to a $\mathrm{CO}$ detection in the shock itself (Gao \& Xu 2000). The single-dish observations by Lisenfeld et al. (2002) using the IRAM 30 m telescope partially overlap the shock structure and

\footnotetext{
11 Berkeley Illinois Maryland Association,

http://bima.astro.umd.edu/bima.html.
}

show a $3 \sigma$ detection of $\mathrm{CO}$ in the northern region of the X-ray ridge, but their observations do not probe the entire shock region. We note that they include this emission in their SQ-A CO flux. Gao \& Xu (2000), Smith \& Struck (2001), and Petitpas \& Taylor (2005) reported CO emission close to NGC 7318b nucleus and from several other regions in the group.

Another interesting feature of SQ is the presence of a CO-rich (Braine et al. 2001; Lisenfeld et al. 2002, 2004; $7 \times 10^{8} M_{\odot}$ ) region, SQ-B, located within the young $\mathrm{H}$ i tidal tail (see Sulentic et al. 2001), to the south of NGC 7319 (outside the field of view of Figure 1). This region is also bright in the mid-IR (Xu et al. 1999; Guillard et al. 2010), H $\alpha$ (Arp 1973), and UV (Xu et al. 2005). The $\mathrm{H}_{2}$-to-H I gas mass ratio of 0.5 indicates that SQ-B is a tidal dwarf galaxy (TDG) candidate, i.e., a small galaxy which is in the process of formation from the material of the NGC 7319's tidal tail (Boquien et al. 2009). The gas metallicity in SQ-A and SQ-B is slightly higher than solar (Xu et al. 2003; Lisenfeld et al. 2004). This is a strong indication that the gas in these regions has been pulled out from the inner part of a galaxy disk (or maybe several disks) by tidal interactions. TDGs will not be further discussed, as we mostly concentrate on the shock, the bridge and SQ-A in this paper.

\section{CO OBSERVATIONS}

\section{1. $C O(1-0)$ and (2-1) with the IRAM 30 m Telescope}

The observations were made on 2009 June 28-30 at the IRAM $30 \mathrm{~m}$ telescope at Pico Veleta, Spain. We used the heterodyne receiver, EMIR ${ }^{12}$ commissioned in 2009 March-April. EMIR provides a bandwidth of $4 \mathrm{GHz}$ in each of the two orthogonal linear polarizations for the 3,2,1.3, and $0.9 \mathrm{~mm}$ atmospheric windows. The four EMIR bands are designated as E090, $\mathrm{E} 150, \mathrm{E} 230$, and E330 according to their approximate center frequencies in $\mathrm{GHz}$. Given the wide range of $\mathrm{CO}$ velocities (6000-6700 $\mathrm{km} \mathrm{s}^{-1}$ ) exhibited by previous observations, and the broad line width of the $\mathrm{S}(1) \mathrm{H}_{2}$ line in the shock, this increase in bandwidth was crucial to our study.

We observed the $\mathrm{CO}(1-0)$ and $\mathrm{CO}(2-1)$ lines at $115.27 \mathrm{GHz}$ and $230.54 \mathrm{GHz}$ by connecting the third and fourth parts of the WILMA backend to the horizontal and vertical polarizations of the E230 band. We focus this paper on the $\mathrm{CO}(1-0)$ results, since we did not sample the $(1-0)$ beam with the $(2-1)$ observations. To increase the redundancy of the $\mathrm{CO}(1-0)$ data, we used in parallel the $4 \mathrm{MHz}$ filter bank with both polarizations connected to the E090 band. The receivers were tuned to $112.86 \mathrm{GHz}$ and $225.72 \mathrm{GHz}$, which corresponds to a recession velocity of $6400 \mathrm{~km} \mathrm{~s}^{-1}$. The observations were done in wobbler switching mode, with a wobbler throw of $120^{\prime \prime}$ in azimuthal direction. This observation mode provides accurate background subtraction and good quality baselines, which is critical for measuring broad lines. Pointing and focus were monitored every $2 \mathrm{hr}$ in stable conditions and every $1 \mathrm{hr}$ during sunrise. The pointing accuracy was $\sim 3^{\prime \prime}$ and the system temperatures were $\approx 150-200 \mathrm{~K}$ at $115 \mathrm{GHz}$ on the $T_{A}^{*}$ scale. The forward efficiency of the telescope was 0.94 and 0.91 at 115 and $230 \mathrm{GHz}$ and the main beam efficiency was 0.78 and 0.58 , respectively. The half-power beam size is $22^{\prime \prime}$ at $115 \mathrm{GHz}$ and $11^{\prime \prime}$ at $230 \mathrm{GHz}$.

The data were reduced with the IRAM GILDAS-CLASS $90^{13}$ software package (version of 2010 December). The lowest

\footnotetext{
12 For details about the Eight MIxer Receiver instrument, see http://www.iram.es/IRAMES/mainWiki/EmirforAstronomers.

13 Grenoble Image and Line Data Analysis System,

http://www.iram.fr/IRAMFR/GILDAS/.
} 
Table 1

CO Observations Log

\begin{tabular}{lccccc}
\hline \hline Position & \multirow{2}{*}{ R.A. (J2000) } & Decl. (J2000) & \multicolumn{3}{c}{ Integration Time (min) } \\
\cline { 4 - 6 } & & & $\mathrm{CO}(1-0)$ & $\mathrm{CO}(2-1)$ & $\mathrm{CO}(3-2)$ \\
\hline SQ-A $^{\mathrm{a}}$ & $22: 35: 58.88$ & $+33: 58: 50.69$ & 122 & 58 & 66 \\
Ridge 1 & $22: 35: 59.85$ & $+33: 58: 16.55$ & 311 & 186 & 80 \\
Ridge 2 & $22: 35: 59.85$ & $+33: 58: 04.00$ & 318 & 249 & $\ldots$ \\
Ridge 3 & $22: 35: 59.73$ & $+33: 57: 37.16$ & 86 & 41 & $\ldots$ \\
Bridge $^{\mathrm{b}}$ & $22: 36: 01.56$ & $+33: 58: 23.30$ & 175 & 57 & $\ldots$
\end{tabular}

Notes. Pointing positions and useful integration times of the CO spectra presented in this paper. The $\mathrm{CO}(1-0)$ and (2-1) lines were observed with the IRAM 30 m telescope and the $\mathrm{CO}(3-2)$ with the APEX telescope.

${ }^{a}$ Starburst region at the northern tip of the SQ ridge.

b Bridge feature that connects the ridge to the AGN NGC 7319.

quality spectra (typically with an rms noise $\sigma \gtrsim 30 \mathrm{mK}$ for a 3 minute on-source integration) were rejected. We subtracted linear baselines. The final spectra presented in this paper are smoothed (with a hanning window) to a velocity resolution of $40 \mathrm{~km} \mathrm{~s}^{-1}$. We experienced problems with the vertical polarization. These spectra, which showed spurious features, ripples, and curved baselines, have been rejected for this analysis.

We observed five different positions in the center of the SQ group. The blue circles on Figure 1 show the $\mathrm{CO}(1-0)$ halfpower beams (22" in diameter) overlaid on a Hubble Space Telescope (HST) WFPC2 image of SQ. The three positions in the ridge are designated as $\mathrm{R} 1, \mathrm{R} 2$, and $\mathrm{R} 3$, from north to south. The two other positions are centered on the SQ-A star-forming region and on the middle of the $\mathrm{H}_{2}$-bridge feature in between NGC 7319 and the main shock. The red lines show the contours of the $\mathrm{H}_{2} \mathrm{O}-0 \mathrm{~S}(3)$ line emission detected by Spitzer (Cluver et al. 2010). Note that the half-power beamwidth at $115 \mathrm{GHz}$ matches very well the width of the $\mathrm{H}_{2}$ emission in the ridge. Table 1 lists the coordinates of these positions and the total ON+OFF time spent at each position. A total of $\approx 20 \mathrm{hr}$ has been spent for $\mathrm{ON}+\mathrm{OFF}$ observations toward SQ.

\section{2. $C O(3-2)$ with the APEX Telescope}

Observations toward the SQ in the $\mathrm{CO}(3-2)$ transition were carried out during night time in 2010 July with the APEX telescope (Güsten et al. 2006), using the First Light APEX Submillimeter Heterodyne receiver (developed by the Max Planck Institut für Radioastronomie, MPIfR, and commissioned in 2010 May) at $345 \mathrm{GHz}$, FLASH345, ${ }^{14}$ along with two newly commissioned MPIfR X-Fast Fourier Transform Spectrometer backends, XFFTS (R. I. Klein et al., in preparation), of $2.5 \mathrm{GHz}$ bandpass each, and whose combination covers a $4 \mathrm{GHz}$ bandpass. The beam of the telescope is $18^{\prime \prime} .1$ at $345 \mathrm{GHz}$ (Güsten et al. 2006). Focus was checked at the beginning of each night, and pointing was checked on planets (Jupiter, Saturn, and/or Uranus), as well as on CRL2688 every 90 minutes at least.

System temperatures were in the range 180-270 K and $180-230 \mathrm{~K}$ in the respective cases of the Ridge 1 and SQ-A positions, on the $T_{A}$ scale. Antenna temperatures were converted to main beam temperatures using forward and beam efficiencies, respectively, of 0.95 and 0.67 at $345 \mathrm{GHz}$. We used 32,768 channels for each XFFTS backends, yielding an effective spectral resolution of about $76.3 \mathrm{kHz}$. The observations were

\footnotetext{
14 In the $345 \mathrm{GHz}$ band the dual-polarization receiver FLASH operates a 2SB SIS mixer provided by IRAM (Maier et al. 2005).
}

performed in wobbler switching mode, with a throw of $120^{\prime \prime}$ in the azimuthal direction.

Again, the data were reduced by means of the IRAM GILDAS software package. We noted that $\approx 15$ channels (out of a total of 32,768 covering the $4800-8400 \mathrm{~km} \mathrm{~s}^{-1}$ velocity range) were affected by spurious spikes, likely due to the electronics of other receivers that were powered-on at the time of the observations). Therefore, a sigma-clipping has been applied. By scanning across all spectra, the sigma associated with each channel, $\sigma_{c}$, is determined. The data are flagged if the signal is greater than $5 \sigma_{c}$ and greater than $3.5 \sigma_{s}$, where $\sigma_{s}$ is the sigma associated with one spectrum, since the signal does not reach this level.

The baseline subtraction was always linear, as we enjoyed very good baseline stability due to excellent weather conditions during the observations. The final spectra shown here are smoothed to a velocity resolution of $40 \mathrm{~km} \mathrm{~s}^{-1}$, for comparisons purposes with the other transitions obtained at the IRAM $30 \mathrm{~m}$ telescope.

\section{KINEMATICS AND EXCITATION OF THE CO GAS}

\subsection{CO Spectra and Analysis}

In Figure 2, we show the $\mathrm{CO}(1-0)$ and $\mathrm{CO}(2-1)$ spectra for each of the five observed positions. The spectra are plotted over a $4000 \mathrm{~km} \mathrm{~s}^{-1}$ bandwidth (out of the $\approx 8000 \mathrm{~km} \mathrm{~s}^{-1}$ bandwidth available at $115 \mathrm{GHz}$ ) at a spectral resolution of $40 \mathrm{~km} \mathrm{~s}^{-1}$. The noise levels in the average spectra are typically $\approx 0.7-1.6 \mathrm{mK}$ for $\mathrm{CO}(1-0)$ and $\approx 2-5 \mathrm{mK}$ for $\mathrm{CO}(2-1)$ at this resolution. The two transitions of $\mathrm{CO}$ are detected with a high signal-tonoise ratio for all the targeted regions, except a $2.5 \sigma$ tentative detection of $\mathrm{CO}(2-1)$ for the R3 position. Such detections are in sharp contrast with the previous interferometric observations, where no $\mathrm{CO}$ was detected in the ridge, probably because of limited velocity coverage and sensitivity. It shows that $\mathrm{CO}$ gas is present in the $\mathrm{H}_{2}$-luminous shock and is cospatial with the $\mathrm{X}$-ray emitting hot plasma in this region, at a $\sim 5 \mathrm{kpc}$ scale.

The spectrum resulting from our pilot study with APEX at the ridge 1 position, where the $\mathrm{CO}(3-2)$ line is detected, is shown in Figure 3. In SQ-A, the line is tentatively detected at a $2.3 \sigma$ significance, and we consider this as an upper limit on the $\mathrm{CO}(3-2)$ flux.

To compute the $\mathrm{CO}$ line parameters, we applied two different methods. First, we decomposed the CO spectra in multiple Gaussian components using the IDL nonlinear least-squares MPFIT routine (Markwardt 2009). The red lines on Figures 2 and 3 show the fitting result, which is used to derive the central velocities of the $\mathrm{CO}$ components, the line widths, and integrated intensities $\mathcal{I}_{\mathrm{CO}}$. We checked that the residuals are below the $2 \sigma$ noise level for all positions and all lines. The results of these Gaussian decompositions are given in Tables 2, 4, and 5.

Second, the CO line intensities $\mathcal{I}_{\mathrm{CO}}$ were derived from the integration of the $\mathrm{CO}$ main beam temperature, $\mathcal{T}_{\mathrm{mb}}[\mathrm{K}]$, over velocity ranges, from $v_{\min }$ to $v_{\max }$, defined for each spectrum in Table 3, as the following:

$$
\mathcal{I}_{\mathrm{CO}}\left[\mathrm{K} \mathrm{km} \mathrm{s}^{-1}\right]=\int_{\langle v\rangle-v_{\min }}^{v_{\max }-\langle v\rangle} \mathcal{T}_{\mathrm{mb}} d v,
$$

where $\langle v\rangle$ is the first moment (mean, intensity-weighted) velocity over that velocity range. This method allows us to better take into account the asymmetry in the $\mathrm{CO}$ profiles. The results of these integrations are given in Table 3 and discussed in the following sections. 

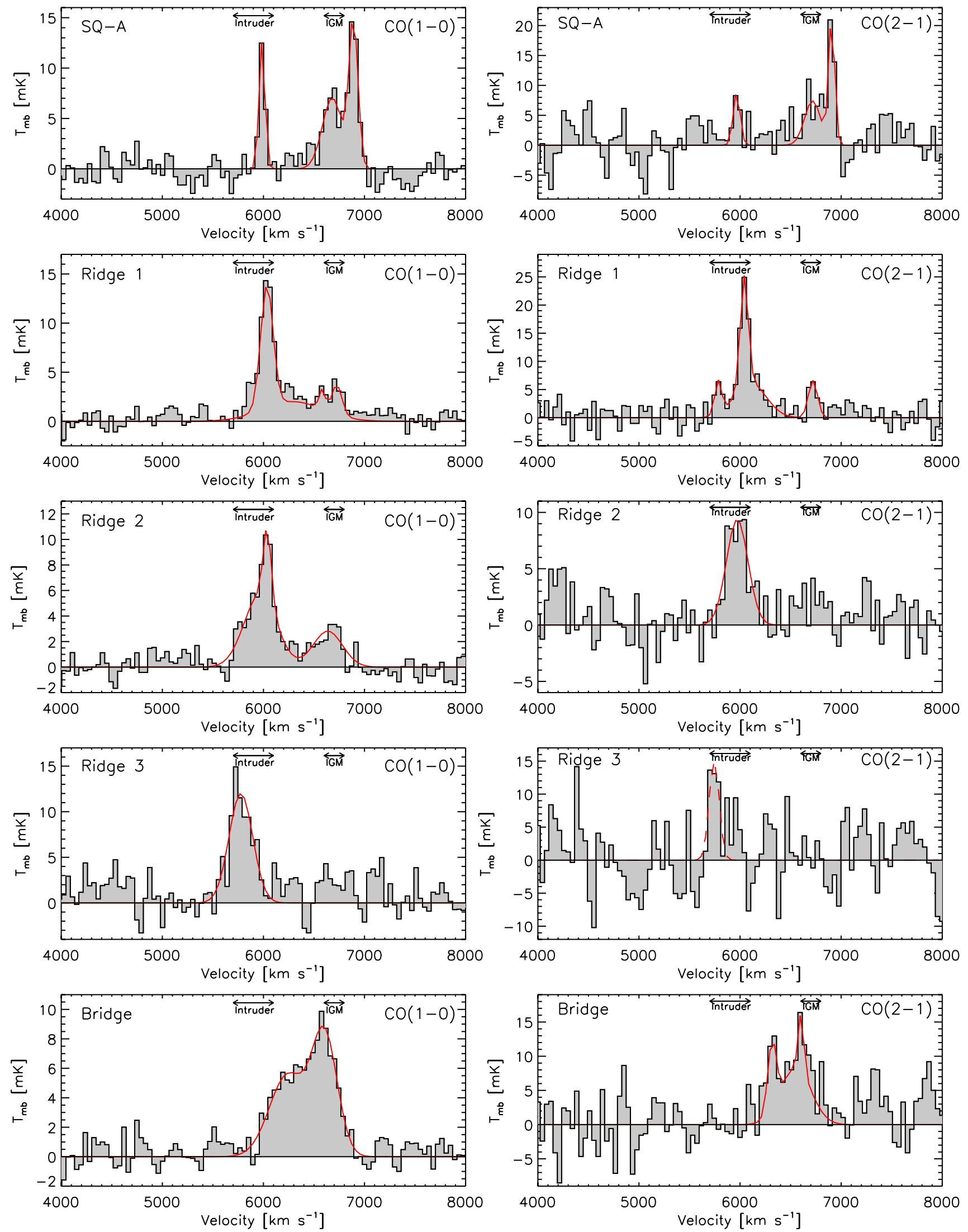

Figure 2. $\mathrm{CO}(1-0)$ and $\mathrm{CO}(2-1)$ spectra (left and right columns) observed with the IRAM $30 \mathrm{~m}$ telescope at the five positions observed in the IGM of the SQ group (see Figure 1). CO emission with multiple velocity components is detected at all positions: in the shock, in SQ-A, and in the bridge regions. The red line shows the result of the line fitting (see Tables 2 and 4 for the decomposition in multiple Gaussian components for each transition). The spectra have been smoothed to a spectral resolution of $40 \mathrm{~km} \mathrm{~s}^{-1}$. Note that the $\mathrm{CO}(1-0)$ and $\mathrm{CO}(2-1)$ beams do not match, and that the detection of the $\mathrm{CO}(2-1)$ line in the Ridge 3 position is tentative (in this case the Gaussian fitting — dashed line-is not reliable). The arrows indicate the pre-shock H I velocities for the intruder galaxy and the intra-group medium.

(A color version of this figure is available in the online journal.) 
Table 2

CO(1-0) Observations with the IRAM 30 m Telescope: Results from Gaussian Decompositions

\begin{tabular}{|c|c|c|c|c|c|c|}
\hline Target Region & $\begin{array}{l}v_{\mathrm{CO}(1-0)} \\
\left(\mathrm{km} \mathrm{s}^{-1}\right)\end{array}$ & $\begin{array}{c}\Delta v_{\mathrm{CO}(1-0)} \\
\left(\mathrm{km} \mathrm{s}^{-1}\right)\end{array}$ & $\begin{array}{c}\mathcal{I}_{\mathrm{CO}(1-0)} \\
\left(\mathrm{K} \mathrm{km} \mathrm{s}^{-1}\right)\end{array}$ & $\begin{array}{c}M\left(\mathrm{H}_{2}\right)^{\mathrm{a}} \\
\left(\times 10^{8} M_{\odot}\right)\end{array}$ & $\begin{array}{l}E_{\text {kin }}{ }^{b} \\
(\text { erg) }\end{array}$ & $M\left(\mathrm{H}_{2}^{\mathrm{w}}\right) / M\left(\mathrm{H}_{2}^{\mathrm{c}}\right)^{\mathrm{c}}$ \\
\hline \multirow[t]{2}{*}{ SQ-A } & $5987 \pm 14$ & $69 \pm 8$ & $0.9 \pm 0.1$ & $3.1 \pm 0.5$ & $7.8 \pm 3.1 \mathrm{E} 54$ & \\
\hline & $6681 \pm 22$ & $230 \pm 35$ & $1.7 \pm 0.3$ & $5.7 \pm 1.1$ & $1.6 \pm 0.8 \mathrm{E} 56$ & \\
\hline \multicolumn{3}{|l|}{ SQ-A (sum) ${ }^{d}$} & $4.2 \pm 0.4$ & $14.1 \pm 1.5$ & $2.0 \pm 0.8$ E56 & $0.9 \pm 0.2$ \\
\hline \multirow[t]{2}{*}{ Ridge 1} & $6034 \pm 11$ & $144 \pm 10$ & $1.9 \pm 0.2$ & $6.3 \pm 0.8$ & $7.1 \pm 1.8 \mathrm{E} 55$ & \\
\hline & $6727 \pm 15$ & $118 \pm 13$ & $0.4 \pm 0.06$ & $1.2 \pm 0.2$ & $9.4 \pm 0.4$ E54 & \\
\hline \multicolumn{3}{|l|}{ Ridge 1 (tot $)^{d}$} & $4.0 \pm 0.3$ & $13.0 \pm 1.4$ & $1.4 \pm 0.4$ E57 & $0.26 \pm 0.03$ \\
\hline \multirow[t]{3}{*}{ Ridge 2} & $5970 \pm 28$ & $377 \pm 36$ & $2.3 \pm 0.3$ & $7.5 \pm 1.1$ & $5.8 \pm 1.9 \mathrm{E} 56$ & \\
\hline & $6038 \pm 12$ & $93 \pm 10$ & $0.56 \pm 0.08$ & $1.9 \pm 0.3$ & $8.6 \pm 3.2 \mathrm{E} 54$ & \\
\hline & $6637 \pm 35$ & $342 \pm 28$ & $1.0 \pm 0.1$ & $3.4 \pm 0.4$ & $2.1 \pm 0.6$ E56 & \\
\hline & $6605 \pm 20$ & $298 \pm 19$ & $2.6 \pm 0.4$ & $8.5 \pm 1.0$ & $4.1 \pm 1.0 \mathrm{E} 56$ & \\
\hline \multicolumn{3}{|l|}{ Bridge (tot) ${ }^{\mathrm{d}}$} & $5.1 \pm 0.5$ & $16.7 \pm 1.4$ & $1.2 \pm 0.2 \mathrm{E} 57$ & $0.55 \pm 0.09$ \\
\hline
\end{tabular}

Notes. Observational results of the IRAM $30 \mathrm{~m}$ EMIR CO(1-0) observations. This table gathers the results from the fitting of the $\mathrm{CO}(1-0)$ spectra in multiple Gaussian velocity components (see Figure 2). The first three columns list the central velocity, FWHM, and intensity of the Gaussian components. Results are given for each velocity components, as well as for the sum of the components. The velocity component at $6900 \mathrm{~km} \mathrm{~s}^{-1}$ in SQ-A is a newly discovered feature (see the text for details). The aperture of the beam is $1.13 \times(22 \operatorname{arcsec})^{2}=547 \operatorname{arcsec}^{2}$.

${ }^{\mathrm{a}} \mathrm{H}_{2}$ gas masses calculated using the Galactic conversion factor of $N\left(\mathrm{H}_{2}\right) / I_{\mathrm{CO}}=2 \times 10^{20} \mathrm{~cm}^{-2}\left[\mathrm{~K} \mathrm{~km} \mathrm{~s}^{-1}\right]^{-1}$.

${ }^{\mathrm{b}}$ Kinetic energy in random motions, calculated as $E_{\mathrm{kin}}=3 / 2 M\left(\mathrm{H}_{2}\right) \sigma_{\mathrm{CO}}^{2}$ (see Section 6.1).

${ }^{\mathrm{c}}$ Ratio between the $\mathrm{H}_{2}$ mass derived from the Spitzer spectral mapping of the mid-IR $\mathrm{H}_{2}$ lines in the group to the $\mathrm{H}_{2}$ mass derived from the $\mathrm{CO}(1-0)$ line intensity at the different positions in the IGM of SQ.

${ }^{\mathrm{d}}$ Sum over all velocity components.

Table 3

CO(1-0) Observations with the IRAM 30 m Telescope: Results from Integrations of the Spectra Over Velocity Ranges

\begin{tabular}{|c|c|c|c|c|c|c|}
\hline Target Region & $\begin{array}{c}\text { Velocity Range } \\
v_{\min }-v_{\max }\left(\mathrm{km} \mathrm{s}^{-1}\right)\end{array}$ & $\begin{array}{c}\langle v\rangle \\
\left(\mathrm{km} \mathrm{s}^{-1}\right)\end{array}$ & $\begin{array}{c}\mathcal{I}_{\mathrm{CO}(1-0)} \\
\left(\mathrm{K} \mathrm{km} \mathrm{s}^{-1}\right)\end{array}$ & $\begin{array}{c}M\left(\mathrm{H}_{2}\right)^{\mathrm{a}} \\
\left(\times 10^{8} M_{\odot}\right)\end{array}$ & $\begin{array}{l}E_{\text {kin }}{ }^{b} \\
\text { (erg) }\end{array}$ & $M\left(\mathrm{H}_{2}^{\mathrm{w}}\right) / M\left(\mathrm{H}_{2}^{\mathrm{c}}\right)^{\mathrm{c}}$ \\
\hline \multirow[t]{4}{*}{ SQ-A } & $5900-6200$ & 5984 & $0.9 \pm 0.1$ & $3.0 \pm 0.2$ & $3.2 \pm 0.8 \mathrm{E} 54$ & \\
\hline & $6200-6500$ & 6375 & $0.4 \pm 0.05$ & $1.3 \pm 0.9$ & $3.5 \pm 0.8$ E55 & \\
\hline & $6500-6800$ & 6676 & $1.5 \pm 0.2$ & $4.8 \pm 0.5$ & $7.4 \pm 1.6$ E55 & \\
\hline & $6800-7100$ & 6882 & $1.7 \pm 0.2$ & $5.6 \pm 0.6$ & $1.7 \pm 0.5$ E55 & \\
\hline SQ-A (sum) ${ }^{\mathrm{d}}$ & & & $4.5 \pm 0.3$ & $14.7 \pm 1.2$ & $1.3 \pm 0.2 \mathrm{E} 56$ & $0.8 \pm 0.2$ \\
\hline \multirow[t]{5}{*}{ Ridge 1} & $5500-5900$ & 5831 & $0.4 \pm 0.05$ & $1.3 \pm 0.3$ & $2.2 \pm 0.6 \mathrm{E} 55$ & \\
\hline & $5900-6200$ & 6041 & $2.5 \pm 0.3$ & $8.3 \pm 1.0$ & $1.1 \pm 0.4$ E56 & \\
\hline & $6200-6500$ & 6325 & $0.8 \pm 0.1$ & $2.5 \pm 0.1$ & $5.5 \pm 0.3 \mathrm{E} 55$ & \\
\hline & $6500-6800$ & 6656 & $0.9 \pm 0.1$ & $2.9 \pm 0.1$ & $6.4 \pm 0.3$ E55 & \\
\hline & $6800-7500$ & 7039 & $0.5 \pm 0.05$ & $1.6 \pm 0.2$ & $1.0 \pm 0.4 \mathrm{E} 56$ & \\
\hline Ridge 1 (sum) ${ }^{\mathrm{d}}$ & & & $5.2 \pm 0.4$ & $16.6 \pm 1.5$ & $3.5 \pm 0.8 \mathrm{E} 56$ & $0.20 \pm 0.03$ \\
\hline \multirow[t]{3}{*}{ Ridge 2} & $5700-6200$ & 5971 & $2.6 \pm 0.3$ & $8.6 \pm 1.2$ & $3.5 \pm 0.8$ E56 & \\
\hline & $6200-6500$ & 6346 & $0.4 \pm 0.05$ & $1.5 \pm 0.3$ & $3.8 \pm 0.9$ E55 & \\
\hline & $6500-6800$ & 6660 & $0.7 \pm 0.1$ & $2.4 \pm 0.3$ & $4.4 \pm 1.0 \mathrm{E} 55$ & \\
\hline Ridge 2 (sum) ${ }^{\mathrm{d}}$ & & & $3.7 \pm 0.4$ & $12.5 \pm 1.5$ & $4.3 \pm 1.1 \mathrm{E} 56$ & $0.34 \pm 0.04$ \\
\hline Ridge 3 & $5500-6100$ & 5788 & $3.5 \pm 0.4$ & $11.6 \pm 1.7$ & $4.5 \pm 0.6$ E56 & $0.10 \pm 0.02$ \\
\hline Bridge & $5900-7000$ & 6435 & $4.9 \pm 0.4$ & $16.0 \pm 2.0$ & $2.4 \pm 0.5 \mathrm{E} 57$ & $0.57 \pm 0.09$ \\
\hline
\end{tabular}

Notes. Idem as Table 2 but for integration of the $\mathrm{CO}(1-0)$ spectra over velocity ranges. The first three columns list the velocity range, the first moment velocity (mean, intensity-weighted velocity), and $\mathrm{CO}(1-0)$ intensity (cf. Equation (1)). Results are given for each velocity ranges, as well as for the sum of the integrations.

${ }^{a} \mathrm{H}_{2}$ gas masses calculated using the Galactic conversion factor of $N\left(\mathrm{H}_{2}\right) / I_{\mathrm{CO}}=2 \times 10^{20} \mathrm{~cm}^{-2}\left[\mathrm{~K} \mathrm{~km} \mathrm{~s}^{-1}\right]^{-1}$.

${ }^{\mathrm{b}}$ Bulk kinetic energy, calculated from Equation (3) (see Section 6.1).

${ }^{c}$ Ratio between the $\mathrm{H}_{2}$ mass derived from the Spitzer spectral mapping of the mid-IR $\mathrm{H}_{2}$ lines in the group to the $\mathrm{H}_{2}$ mass derived from the $\mathrm{CO}(1-0)$ line intensity at the different positions in the IGM of SQ.

${ }^{\mathrm{d}}$ Sum over all velocity ranges at a given position. 


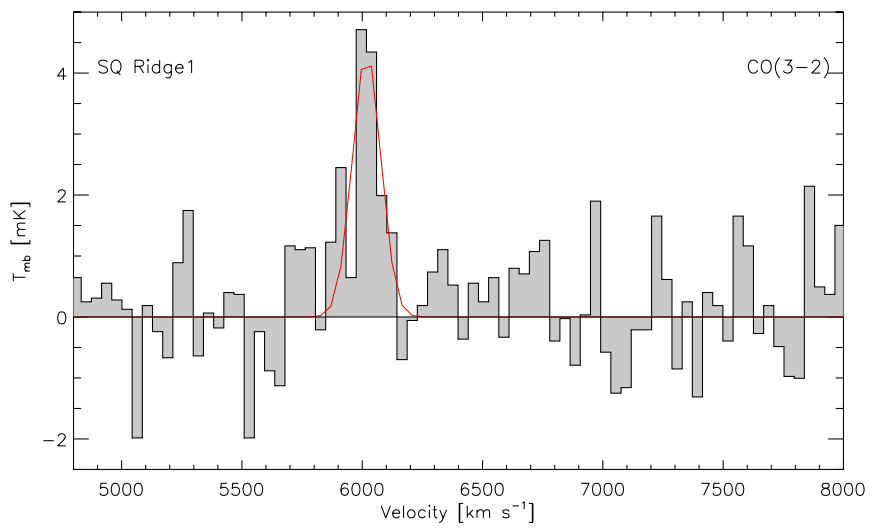

Figure 3. $\mathrm{CO}(3-2)$ spectra observed with APEX at the position Ridge 1 in the IGM of the SQ group (see Figure 1). The red line shows the result of the line fitting (see Table 5). The spectrum has been smoothed to a spectral resolution of $40 \mathrm{~km} \mathrm{~s}^{-1}$.

(A color version of this figure is available in the online journal.)

Table 4

$\mathrm{CO}(2-1)$ Observations with the IRAM 30 m Telescope: Results from Gaussian Decompositions

\begin{tabular}{lccc}
\hline \hline Target Region & $\begin{array}{c}v_{\mathrm{CO}(2-1)} \\
\left(\mathrm{km} \mathrm{s}^{-1}\right)\end{array}$ & $\begin{array}{c}\Delta v_{\mathrm{CO}(2-1)} \\
\left(\mathrm{km} \mathrm{s}^{-1}\right)\end{array}$ & $\begin{array}{c}\mathcal{I}_{\mathrm{CO}(2-1)} \\
\left(\mathrm{K} \mathrm{km} \mathrm{s}^{-1}\right)\end{array}$ \\
\hline SQ-A & $5966 \pm 25$ & $81 \pm 8$ & $0.76 \pm 0.11$ \\
& $6719 \pm 36$ & $292 \pm 35$ & $2.3 \pm 0.4$ \\
& $6906 \pm 14$ & $62 \pm 12$ & $1.4 \pm 0.2$ \\
\hline SQ-A (tot) & & $80 \pm 10$ & $4.5 \pm 0.3$ \\
\hline Ridge 1 & $5783 \pm 14$ & $92 \pm 12$ & $0.50 \pm 0.08$ \\
& $6041 \pm 16$ & $367 \pm 34$ & $1.9 \pm 0.3$ \\
& $6101 \pm 39$ & $105 \pm 13$ & $0.74 \pm 0.4$ \\
\hline Ridge 1 (tot) & $6721 \pm 12$ & & $5.5 \pm 0.5$ \\
\hline Ridge 2 & & $252 \pm 36$ & $2.5 \pm 0.5$ \\
\hline Ridge 3 & $5972 \pm 28$ & $117 \pm 19$ & $1.9 \pm 0.4$ \\
\hline Bridge & $5743 \pm 25$ & $77 \pm 12$ & $0.7 \pm 0.1$ \\
& $6318 \pm 22$ & $648 \pm 36$ & $5.2 \pm 1.6$ \\
\hline Bridge (tot) & $6552 \pm 46$ & $91 \pm 11$ & $0.8 \pm 0.1$ \\
\hline & $6606 \pm 24$ & $6.7 \pm 1.6$ \\
\hline
\end{tabular}

Notes. Idem as Table 2 but for the IRAM 30 m EMIR CO(2-1) observations. The size of the $\mathrm{CO}(2-1)$ beam is $1.13 \times(11 \operatorname{arcsec})^{2}=137 \operatorname{arcsec}^{2}$.

\subsection{Multiple CO Velocity Components}

The spectra in Figures 2 and 3 clearly show that multiple, broad velocity components are detected, pointing out the complexity of the kinematics of the CO gas in the SQ group. The CO line emission extends over a very wide velocity range $\approx 5700-7000 \mathrm{~km} \mathrm{~s}^{-1}$ (and perhaps until $7400 \mathrm{~km} \mathrm{~s}^{-1}$ for the ridge 1 position). The four main velocity components are at 5700, 6000, 6700, and $6900 \mathrm{~km} \mathrm{~s}^{-1}$ (see Tables 2, 4, and 5 for the central line velocities derived from line fitting). The gas associated with the intruder NGC $7318 \mathrm{~b}\left(5700-6000 \mathrm{~km} \mathrm{~s}^{-1}\right)$ and with the intra-group medium $\left(6700 \mathrm{~km} \mathrm{~s}^{-1}\right)$ are both detected in the ridge and in the eastern feature (the bridge) toward NGC 7319 as well. These two CO velocity systems match with the velocities of the H I gas observed in the group (Sulentic et al. 2001; Williams et al. 2002). In Ridge 3, the southern part of the shock, most of the gas seems to be associated with the intruder, since the $6700 \mathrm{~km} \mathrm{~s}^{-1}$ component is not detected. The velocity

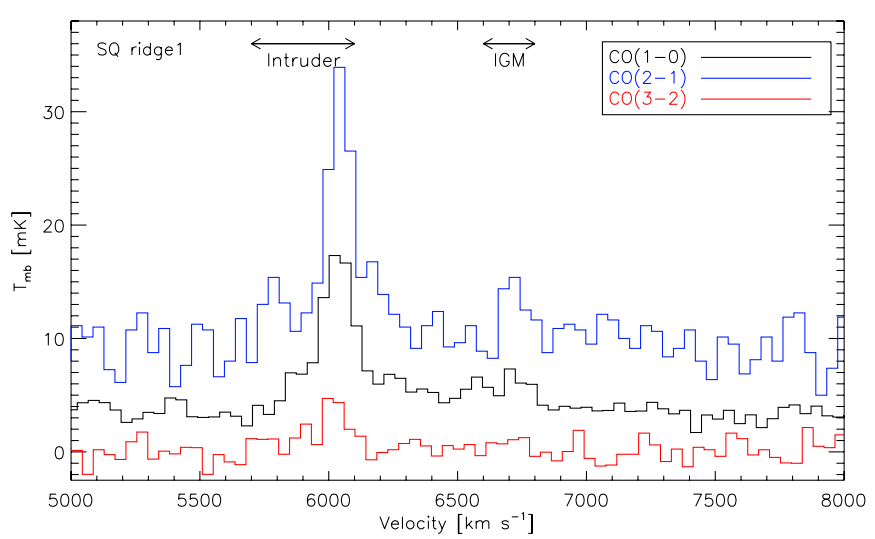

Figure 4. Overlay of the $\mathrm{CO}(1-0), \mathrm{CO}(2-1)$, and $\mathrm{CO}(3-2)$ spectra observed in the Stephan's Quintet shock, at the positions Ridge 1. No beam size convolution has been applied. The spectra have been shifted vertically for clarity. The arrows indicate the pre-shock H I velocities for the intruder galaxy and the intra-group medium.

(A color version of this figure is available in the online journal.)

Table 5

$\mathrm{CO}(3-2)$ Observations with APEX: Results

\begin{tabular}{lcrc}
\hline \hline Target & $\begin{array}{c}v_{\mathrm{CO}(3-2)} \\
\left(\mathrm{km} \mathrm{s}^{-1}\right)\end{array}$ & $\begin{array}{r}\Delta v_{\mathrm{CO}(3-2)} \\
\left(\mathrm{km} \mathrm{s}^{-1}\right)\end{array}$ & $\begin{array}{c}\mathcal{I}_{\mathrm{CO}(3-2)} \\
\left(\mathrm{K} \mathrm{km} \mathrm{s}^{-1}\right)\end{array}$ \\
\hline SQ-A $^{\mathrm{a}}$ & $6858 \pm 66$ & $80 \pm 31$ & $0.32 \pm 0.12$ \\
Ridge 1 & $5976 \pm 15$ & $134 \pm 43$ & $0.65 \pm 0.13$ \\
\hline
\end{tabular}

Notes. Idem as Tables 2 and 4 but for the APEX CO(3-2) observations (see Figure 3) obtained with the FLASH345/XFFTS receivers. The aperture of the $\mathrm{CO}(3-2)$ beam is $1.13 \times(18.1 \mathrm{arcsec})^{2} \approx 370 \operatorname{arcsec}^{2}$.

a Tentative detection at a $2.3 \sigma$ level.

component at $6900 \mathrm{~km} \mathrm{~s}^{-1}$ is a new feature that we detect only in the SQ-A region. It was not seen in previous $\mathrm{CO}$ or $\mathrm{H}$ I observations because of limited velocity coverage, and its origin is still unknown. It could be the result of dynamical effects during the previous encounter between NGC 7319 and 7320c, but current hydrodynamical simulations cannot reproduce this feature. We note that an $\mathrm{H} \alpha$ velocity component at $7000 \mathrm{~km} \mathrm{~s}^{-1}$ was reported by Sulentic et al. (2001) in a clump to the northeast of SQ-A, but it is outside of our $\mathrm{CO}(1-0)$ beam.

Gas at intermediate velocities, in between that of the intruder and that of the intra-group, is detected. A weak, broad component centered at $\approx 6400 \mathrm{~km} \mathrm{~s}^{-1}$ is detected in the ridges 1 and 2 , and in the bridge positions. Interestingly, this intermediate component seems to be absent (or very weak, at $\mathrm{a} \approx 2 \sigma$ significance) in SQ-A and vanishes at the southern end of the main shock region (ridge 3 ). The $6400 \mathrm{~km} \mathrm{~s}^{-1} \mathrm{CO}$ velocity component is consistent with the central velocity of the broad, resolved $\mathrm{H}_{2} \mathrm{~S}(1)$ line at $6360 \pm 100 \mathrm{~km} \mathrm{~s}^{-1}$ (see Appleton et al. 2006; Cluver et al. 2010 for an analysis of the high-resolution Spitzer IRS spectrum). This is the first time $\mathrm{CO}$ gas is detected at these intermediate velocities, and this gas is spatially associated with the warm $\mathrm{H}_{2}$ seen by Spitzer. Xu et al. (2003) and IglesiasPáramo et al. (2012) reported that the low velocity component (intruder) of the optical emission lines in the shock and in the bridge is centered at intermediate velocities (between 5900 and $6500 \mathrm{~km} \mathrm{~s}^{-1}$ ). In the northern (SQ-A) and southern star-forming regions, at both ends of the large-scale shock, the low velocity component of the optical lines is consistent with the intruder velocity.

In Figure 4, we compare the three $\mathrm{CO}$ line profiles at the ridge 1 and SQ-A positions. At both positions, the central velocity of 
Table 6

$\mathrm{H}_{2}$ Rotational Line Intensities

\begin{tabular}{|c|c|c|c|c|c|c|}
\hline Target Region & $\mathrm{H}_{2} \mathrm{~S}(0)$ & $\mathrm{H}_{2} \mathrm{~S}(1)$ & $\mathrm{H}_{2} \mathrm{~S}(2)$ & $\mathrm{H}_{2} \mathrm{~S}(3)$ & $\mathrm{H}_{2} \mathrm{~S}(4)$ & $\mathrm{H}_{2} \mathrm{~S}(5)$ \\
\hline SQ-A & $1.47 \pm 0.11$ & $6.26 \pm 0.13$ & & & & \\
\hline ridge 1 & $0.79 \pm 0.04$ & $11.0 \pm 1.56$ & $3.19 \pm 0.25$ & $11.5 \pm 1.31$ & $1.97 \pm 0.27$ & $4.97 \pm 0.41$ \\
\hline Ridge 2 & $0.68 \pm 0.04$ & $7.06 \pm 0.42$ & $2.75 \pm 0.11$ & $13.3 \pm 2.35$ & $1.78 \pm 0.33$ & $4.31 \pm 0.31$ \\
\hline Ridge 3 & $0.49 \pm 0.07$ & $9.55 \pm 1.51$ & $3.42 \pm 0.31$ & $5.52 \pm 1.53$ & $0.94 \pm 0.34$ & $2.85 \pm 0.39$ \\
\hline Bridge & $0.88 \pm 0.07$ & $4.88 \pm 0.08$ & & & & \\
\hline
\end{tabular}

Notes. $\mathrm{H}_{2}$ line fluxes derived from the Spitzer IRS spectra extracted at the positions of our CO observations. Fluxes are in units of $10^{-17} \mathrm{~W}$ m ${ }^{-2}$.

the $\mathrm{CO}(3-2)$ line coincide with the brightest $\mathrm{CO}(1-0)$ and (2-1) components. The FWHM of the $\mathrm{CO}(3-2)$ line matches those of the brighter $\mathrm{CO}(1-0)$ and (2-1) lines.

\subsection{High $C O$ Velocity Dispersions}

In the ridge, the FWHM of the main CO components are of the order of 100-400 $\mathrm{km} \mathrm{s}^{-1}$ (see Tables 2, 4, and 5). In the ridge 1 position (and perhaps ridge 2), the underlying intermediate component at $6400 \mathrm{~km} \mathrm{~s}^{-1}$ is extremely broad, $\approx 1000 \mathrm{~km} \mathrm{~s}^{-1}$, with possibly a hint of emission between 6900 and $7400 \mathrm{~km} \mathrm{~s}^{-1}$. This CO velocity coverage of $\approx 1000 \mathrm{~km} \mathrm{~s}^{-1}$ is comparable to the relative velocity between the intruder and the IGM, and to the broadening of the $\mathrm{H}_{2} \mathrm{~S}(1)$ line $\left(\mathrm{FWHM} \approx 870 \mathrm{~km} \mathrm{~s}^{-1}\right.$ ) observed by Appleton et al. (2006) and Cluver et al. (2010).

In $S Q-A$, the line widths are of the order of $100 \mathrm{~km} \mathrm{~s}^{-1}$, with the smallest dispersion for the $6000 \mathrm{~km} \mathrm{~s}^{-1}$ line. The broadest component at $6700 \mathrm{~km} \mathrm{~s}^{-1}$ seems to have a left wing extending toward $6400 \mathrm{~km} \mathrm{~s}^{-1}$, suggesting again that some of the CO gas belonging to the IGM has been decelerated in the shock induced by the intruder galaxy. Interestingly, for the three transitions, the CO line widths are significantly smaller in SQ-A than in the ridge and bridge, and this is perhaps a clue to understand why there is much more star formation in this region than in the ridge (see the discussion in Section 7.1). This observational picture fits within the interpretation of two colliding gas flows, where the shear velocities between the flows are maximum in the central region of the contact discontinuity (ridge), and smaller at the edges of the main shock structure (see Figure 5 of Lee et al. 1996 and Guillard 2010).

The spectrum in the bridge is perhaps the most striking. It shows a very broad signal in between the extreme velocities detected in SQ-A (6000 and $6900 \mathrm{~km} \mathrm{~s}^{-1}$ ), see Figure 2. The $\mathrm{CO}(1-0)$ profile is well fitted by a sum of two Gaussians, whereas in the $\mathrm{CO}(2-1)$ spectrum, narrower velocity components (centered at 6300 and $6600 \mathrm{~km} \mathrm{~s}^{-1}$ ) emerge on top of a broader $\left(\mathrm{FWHM} \approx 650 \mathrm{~km} \mathrm{~s}^{-1}\right)$ emission.

\subsection{CO Excitation}

Since the beams of the $\mathrm{CO}(1-0),(2-1)$, and (3-2) observations do not match, and given that the angular size of the $\mathrm{CO}$ emitting regions are not constrained, we cannot infer useful information on the $\mathrm{CO}$ excitation (from the $\mathrm{CO}$ line ratios). We generally find a $\mathrm{CO}(2-1)$ to $\mathrm{CO}(1-0)$ line ratio of $\approx 1$, which suggests that the $\mathrm{CO}$ is not far from being optically thick. This has to be confirmed with a complete $\mathrm{CO}(2-1)$ mapping of SQ.

\section{DISTRIBUTION AND MASS OF MOLECULAR GAS}

\subsection{Warm $\mathrm{H}_{2}$ Masses from the Spitzer Mid-IR Spectroscopy}

To derive the warm $(T \gtrsim 100 \mathrm{~K}) \mathrm{H}_{2}$ masses from the Spitzer observations, we extracted IRS spectra within the areas observed
Table 7

$\mathrm{H}_{2}$ Line Flux Fitting with MHD Shock Models: Results

\begin{tabular}{lcccc}
\hline \hline Target Region & $\begin{array}{c}\text { Shock Velocities } \\
\left(\mathrm{km} \mathrm{s}^{-1}\right)\end{array}$ & $\begin{array}{c}\text { Mass Flow } \\
\left(M_{\odot} \mathrm{yr}^{-1}\right)\end{array}$ & $\begin{array}{c}\text { Cooling Time } \\
(\mathrm{yr})\end{array}$ & $\begin{array}{c}M\left(\mathrm{H}_{2}^{\text {warm }}\right) \\
\left(M_{\odot}\right)\end{array}$ \\
\hline SQ-A & 5 & $1.47 \times 10^{4}$ & $8.09 \times 10^{4}$ & $1.19 \times 10^{9}$ \\
\hline Ridge 1 & 8 & $6.28 \times 10^{3}$ & $5.33 \times 10^{4}$ & $3.35 \times 10^{8}$ \\
\hline Ridge 2 & 35 & $5.45 \times 10^{2}$ & $9.77 \times 10^{3}$ & $5.33 \times 10^{6}$ \\
\hline Ridge 3 & 6 & $6.61 \times 10^{3}$ & $6.30 \times 10^{4}$ & $4.16 \times 10^{8}$ \\
& 35 & $5.40 \times 10^{2}$ & $9.77 \times 10^{3}$ & $5.27 \times 10^{6}$ \\
\hline Bridge & 13 & $3.32 \times 10^{3}$ & $5.52 \times 10^{4}$ & $1.17 \times 10^{8}$ \\
& 40 & $1.74 \times 10^{2}$ & $7.76 \times 10^{3}$ & $1.35 \times 10^{6}$ \\
\hline
\end{tabular}

Notes. $\mathrm{H}_{2}$ line fluxes are fitted with low-velocity MHD shocks in dense molecular gas for a pre-shock density of $n_{\mathrm{H}}=10^{3} \mathrm{~cm}^{-3}$ and a pre-shock magnetic field of $30 \mu \mathrm{G}$. The table gives the best-fit shock velocities, mass flows, cooling times, and warm $\mathrm{H}_{2}$ masses.

in $\mathrm{CO}(1-0)$ with the CUBISM software (Smith et al. 2007a). The $\mathrm{H}_{2}$ line fluxes, listed in Table 6, were derived from Gaussian fitting with the PAHFIT IDL tool (Smith et al. 2007b). The $\mathrm{H}_{2}$ physical parameters are estimated with the method described in Guillard et al. (2009). The SED of the $\mathrm{H}_{2}$ line emission is modeled with magnetic shocks, using the MHD code described in Flower \& Pineau des Forêts (2010). The gas is heated to a range of post-shock temperatures that depend on the shock velocity, the pre-shock density, and the intensity of the transverse magnetic field. We use a grid of shock models (varying shock speeds) similar to that described in Guillard et al. (2009), to constrain the physical conditions (density and shock velocity) needed to fit the $\mathrm{H}_{2}$ line fluxes. These fits are not unique, and in principle, the observed $\mathrm{H}_{2}$ excitation arises from a continuous distribution of densities and shock velocities. We find a best fit for a pre-shock density $n_{\mathrm{H}}=10^{3} \mathrm{~cm}^{-3}$. The initial orthoto-para ratio is set to 3 , and the intensity of the pre-shock magnetic field is set to $B_{0}=\sqrt{n_{\mathrm{H}}} \approx 30 \mu \mathrm{G}$, in agreement with Zeeman effect observations of Galactic molecular clouds (Crutcher 1999), and the value inferred from radio observations of the synchrotron emission in the SQ ridge (Xu et al. 2003; O'Sullivan et al. 2009b). The $\mathrm{H}_{2}$ line fluxes and the warm $\mathrm{H}_{2}$ masses are computed when the post-shock gas has cooled down to a temperature of $100 \mathrm{~K}$. This temperature is chosen because below $\approx 100 \mathrm{~K}$, the gas does not contribute significantly to the rotational $\mathrm{H}_{2}$ emission. The warm $\mathrm{H}_{2}$ masses derived from the shock models depend on this temperature, the lower the temperature, the longer the cooling time, hence the larger $\mathrm{H}_{2}$ mass. However, the relative difference in cooling times (hence in $\mathrm{H}_{2}$ masses) between $100 \mathrm{~K}$ and $150 \mathrm{~K}$ is $<20 \%$, so this choice of temperature does not impact much the results given in Table 7. 
A combination of two shocks with distinct velocities is required to match the observed $\mathrm{H}_{2}$ line fluxes. As discussed in Guillard et al. (2009), the $\mathrm{H}_{2}$ masses are derived by multiplying the gas cooling time (down to $100 \mathrm{~K}$ ) by the gas mass flow (the mass of gas swept by the shock per unit time) required to match the $\mathrm{H}_{2}$ line fluxes. The shock model parameters, gas cooling times, mass flows, and warm $\mathrm{H}_{2}$ masses are quoted in Table 7. The IRS spectra in different regions of the shock and a detailed description of the spatial variations of $\mathrm{H}_{2}$ excitation (full two-dimensional excitation diagrams) in the SQ intra-group medium will be given in a separate paper (P. N. Appleton et al., in preparation).

\section{2. $\mathrm{H}_{2}$ Masses Derived from $\mathrm{CO}$ Observations}

We derive the masses of $\mathrm{H}_{2}$ gas from the $\mathrm{CO}(1-0)$ line observations, using the Galactic conversion factor $X=$ $N\left(\mathrm{H}_{2}\right) / \mathcal{I}_{\mathrm{CO}}=2 \times 10^{20} \mathrm{~cm}^{-2}\left(\mathrm{~K} \mathrm{~km} \mathrm{~s}^{-1}\right)^{-1}$ :

$$
\begin{aligned}
M_{\mathrm{H}_{2}} & =\mathcal{I}_{\mathrm{CO}}^{\mathrm{obs}} \times \frac{N\left(\mathrm{H}_{2}\right)}{\mathcal{I}_{\mathrm{CO}}} \times d^{2} \times \Omega \times 2 m_{\mathrm{H}} \\
\frac{M_{\mathrm{H}_{2}}}{M_{\odot}} & =3.3 \times 10^{8} \frac{\mathcal{I}_{\mathrm{CO}}^{\mathrm{obs}}}{\mathrm{K} \mathrm{km} \mathrm{s}^{-1}} \frac{N\left(\mathrm{H}_{2}\right) / \mathcal{I}_{\mathrm{CO}}}{2 \times 10^{20}}\left(\frac{d}{94 \mathrm{Mpc}}\right)^{2}\left(\frac{\theta_{\mathrm{HP}}}{22^{\prime \prime}}\right)^{2},
\end{aligned}
$$

where $\mathcal{I}_{\mathrm{CO}}^{\text {obs }}$ is the observed velocity integrated $\mathrm{CO}(1-0)$ line intensity, $d$ is the distance to the source, and $\Omega$ is the area covered by the observations in $\operatorname{arcsec}^{2}$, with $\Omega=1.13 \times \theta_{\mathrm{HP}}^{2}$ for a single pointing with a Gaussian beam of half-power beamwidth of $\theta_{\mathrm{HP}}^{2}$. To derive the total mass of molecular gas, one has to multiply $M_{\mathrm{H}_{2}}$ by a factor 1.36 to take the helium contribution into account. Tables 2 and 3 give the $\mathrm{H}_{2}$ gas masses for the different positions, derived from the $\mathrm{CO}$ line intensities. In the following, we discuss the masses of molecular gas detected in the three main areas, the ridge, SQ-A, and the bridge.

In the $\mathrm{SQ}$ ridge (positions $\mathrm{R} 1, \mathrm{R} 2$, and $\mathrm{R} 3$ ), summing over all the $\mathrm{CO}(1-0)$ velocity components, and assuming a Galactic $\mathrm{CO}$ to $\mathrm{H}_{2}$ conversion factor, we derive a mass of $\approx 4.1 \times 10^{9} M_{\odot}$ of $\mathrm{H}_{2}$ gas, corresponding to a surface density of $\Sigma\left(\mathrm{H}_{2}\right)=12.0 \pm 1.3 M_{\odot} \mathrm{pc}^{-2}$. Note that these three pointings cover $\approx 75 \%$ of the whole $\mathrm{H}_{2}$-bright ridge (the $\mathrm{R} 1$ and $\mathrm{R} 2$ beams are partially overlapping).

Within the $\mathrm{CO}(1-0)$ aperture $\Omega=1.13 \times(22 \text { arcsec })^{2}=$ $547 \operatorname{arcsec}^{2}$ centered on $S Q-A$, we find a total $\mathrm{H}_{2}$ mass of $1.3 \times$ $10^{9} M_{\odot}$. Lisenfeld et al. (2002) find $\approx 3 \times 10^{9} M_{\odot}$ over a much larger area $\left(60 \times 80 \operatorname{arcsec}^{2}\right)$ that partially overlaps the north of the ridge. However, because of a limited bandwidth, they only detected the 6000 and $6700 \mathrm{~km} \mathrm{~s}^{-1}$ velocity components, and not the higher velocity component at $6900 \mathrm{~km} \mathrm{~s}^{-1}$ that contains almost half of the mass in the core region of SQ-A (see Table 2). Based on the MIPS $24 \mu \mathrm{m}$ map, we estimate the area of SQ-A to be $\approx 40 \times 40 \operatorname{arcsec}^{2}$. If we scale the mass we find in our aperture $\Omega$ to this larger aperture, we find a total mass of $\mathrm{H}_{2}$ of $3.8 \times 10^{9} M_{\odot}$. This is comparable to the mass derived by Lisenfeld et al. (2002), because the larger aperture used to estimate their masses compensates their non-detection of the $6900 \mathrm{~km} \mathrm{~s}^{-1}$ component.

In NGC 7319's bridge, summing over all velocities, we find $M_{\mathrm{H}_{2}} \approx 1.7 \times 10^{9} M_{\odot}$ within the aperture $\Omega$. Note that this aperture matches quite well the width of the bridge, but does not cover its whole extension toward the AGN. Therefore, the total mass of molecular gas in the bridge may be a factor of $\approx 2$ larger. Smith \& Struck (2001) find $\approx 5 \times 10^{9} M_{\odot}$ within a half-power beam of 55 arcsec in diameter centered on the nucleus of NGC 7319. This beam overlaps our bridge region. The global morphology of the CO gas in SQ is still unknown, but it is surely linked to the geometry of the collision and the morphology of the colliding gas flows. For instance, the origin of the molecular gas in the bridge structure is still an open question. It is most likely a result of a previous tidal interaction (with NGC 7320c for instance) that would have stripped some material from NGC 7319's galactic disk. Then the interaction between the new intruder and this "tidal bridge" may trigger further molecular gas formation in this region, since the proportion of post-shock gas (with respect to the pre-shock) in this region is the highest observed. Aoki et al. (1996) also reported from optical spectroscopy that an outflow from the AGN is present, which seems oriented in the direction of the bridge feature. Some of the molecular material present in the bridge may be gas entrained from NGC 7319's disk by the outflow. However, it is unlikely that the outflowing ionized gas can entrain the dense molecular gas on such large distances ( $\gtrsim 10 \mathrm{kpc})$.

These observations represent a substantial revision of the mass and energy budgets of the collision. Previous interferometric observations may have missed the molecular gas in the ridge because of limited sensitivity and bandpass and because the $\mathrm{CO}$ emission in the ridge may be more diffuse than in SQ-A. We detect at least four times more $\mathrm{CO}$ emission in the SQ ridge and in the bridge than in the starburst region SQ-A. Although these observations do not cover all the IGM, the CO emission seems distributed over a $40 \mathrm{kpc}$ scale along the ridge, and in total (SQ-A + ridge + bridge), $\gtrsim 5 \times 10^{9} M_{\odot}$ of $\mathrm{H}_{2}$ gas is lying outside the optical disks of the group galaxies. Note that these masses depend on the $X$-factor, which is poorly constrained (Section 5.3).

\subsection{Uncertainties in the $\mathrm{H}_{2}$ Masses}

We computed the uncertainties in the warm $\mathrm{H}_{2}$ masses derived from the Spitzer spectroscopy by fitting shock models to the lower and upper values of the $\mathrm{H}_{2}$ line fluxes, and we found uncertainties on the $\mathrm{H}_{2}$ masses of the order of $20 \%$ (based solely on the observational uncertainties). The warm $\mathrm{H}_{2}$ masses derived from shock models are 10\%-35\% larger than local thermal equilibrium models (we performed single temperature fits for SQ-A and the Bridge regions, and two temperatures fits for the positions in the ridge). This is mainly because (1) the values of the $\mathrm{H}_{2}$ ortho-to-para ratio are different in the two models, (2) at $n_{\mathrm{H}}=10^{3} \mathrm{~cm}^{-3}$, the $\mathrm{S}(0)$ and $\mathrm{S}(1)$ lines are not fully thermalized, and (3) the $\mathrm{H}_{2}$ mass derived from shock models is dependent on the final temperature at which the $\mathrm{H}_{2}$ level populations are calculated.

We derived $\mathrm{H}_{2}$ masses from the $\mathrm{CO}(1-0)$ line intensities using the Galactic value of the CO-to- $\mathrm{H}_{2}$ mass conversion factor (the $X$-factor), because the metallicity in the IGM is slightly above the solar value (Xu et al. 2003). However, the $X$-factor is uncertain, essentially because it is poorly constrained in shocked environments (Downes \& Solomon 1998; Gao et al. 2003), and more generally in low-density molecular gas. For instance, in a similar situation in the bridge between the Taffy galaxies (UGC 12914 and 12915), Braine et al. (2003) estimated an $X$-factor roughly five times below the Galactic value using ${ }^{13} \mathrm{CO}$ measurements. The dust emission modeling by Natale et al. (2010), using their Spitzer/MIPS 70 and $160 \mu \mathrm{m}$ measurements in the shock, gives $\Sigma\left(\mathrm{H}_{2}\right)=9.8 M_{\odot}$ $\mathrm{pc}^{-2}$ (assuming a Galactic dust-to-gas mass ratio $Z_{\mathrm{d}}=0.0075$ ). This value is close $(\approx 20 \%$ smaller $)$ to the average $\mathrm{H}_{2}$ mass 
surface density derived from the $\mathrm{CO}$ measurements in the ridge $\left(\Sigma\left(\mathrm{H}_{2}\right)=12.0 \pm 1.3 M_{\odot} \mathrm{pc}^{-2}\right)$, assuming a Galactic conversion factor (Table 2). This is within the uncertainties of the derived values from the dust modeling. The large uncertainties on the far-IR photometry in the shock are dominated by the difficulty to spatially separate the far-IR emission of the ridge itself from the bright sources surrounding the shock. Upcoming Herschel observations will help in estimating the $X$-factor in the shock and in the surrounding star-forming regions. We defer this discussion to a future paper. The uncertainty on the $X$-factor affects the mass and energy budget discussed in Section 7.3 but does not change the general conclusions of this paper.

\section{ENERGETICS AND EXCITATION OF THE MOLECULAR GAS}

\subsection{Kinetic Energy Carried by the Molecular Gas}

A first estimate of the kinetic energy of the molecular gas was based on Spitzer/IRS spectroscopy of the $\mathrm{H}_{2}$ line emission from SQ (Guillard et al. 2009). Assuming that the $8.8 \times 10^{8} M_{\odot}$ of warm $\mathrm{H}_{2}$ gas detected by Spitzer has a velocity dispersion of $370 \mathrm{~km} \mathrm{~s}^{-1}$ (Cluver et al. 2010), the turbulent kinetic energy carried by the warm $\mathrm{H}_{2}$ gas is $\approx 3.6 \times 10^{57} \mathrm{erg}$. Given the limited spectral resolution of Spitzer/IRS $\left(\approx 600 \mathrm{~km} \mathrm{~s}^{-1}\right)$, this estimate was crude. The present $\mathrm{CO}$ observations allows us to compute the kinetic energy more accurately.

As for the $\mathrm{CO}$ fluxes and masses, we estimate the kinetic energy carried the $\mathrm{CO}$ emitting gas with two methods. First, from the Gaussian decompositions, the turbulent kinetic energy of the $\mathrm{H}_{2}$ gas is derived from $E_{\text {kin }}=3 / 2 M_{\mathrm{H}_{2}} \sigma_{\mathrm{CO}}^{2}$, where $M_{\mathrm{H}_{2}}$ is the $\mathrm{H}_{2}$ mass derived from the $\mathrm{CO}(1-0)$ flux (Equation (2)) and $\sigma_{\mathrm{CO}}$ is the velocity dispersion along the line of sight (measured on the Gaussian fits of the $\mathrm{CO}(1-0)$ line). The factor of three in $E_{\text {kin }}$ takes into account the three dimensions. These kinetic energies are listed in Table 2 for each of the $\mathrm{CO}$ velocity component and each observed positions.

The second method consists in integrating $\mathcal{T}_{\mathrm{mb}} \times(v-\langle v\rangle)^{2}$ over velocity ranges, from $v_{\min }$ to $v_{\max }$, defined for each spectrum and each velocity components in Table 3 :

$$
\begin{aligned}
E_{\mathrm{kin}}= & \frac{3}{2} \times 3.3 \times 10^{8} M_{\odot} \frac{N\left(\mathrm{H}_{2}\right) / \mathcal{I}_{\mathrm{CO}}}{2 \times 10^{20}}\left(\frac{d}{94 \mathrm{Mpc}}\right)^{2}\left(\frac{\theta_{\mathrm{HP}}}{22^{\prime \prime}}\right)^{2} \\
& \times \int_{\langle v\rangle-v_{\min }}^{v_{\max }-\langle v\rangle}(v-\langle v\rangle)^{2} \times \mathcal{T}_{m b} d v
\end{aligned}
$$

where $\langle v\rangle$ is the mean, intensity-weighted (first moment) velocity. The comparison of Tables 2 and 3 shows that the two methods give similar results, essentially because we defined velocity ranges that bracket the main peaks of $\mathrm{CO}$ emission. Integrations over these velocity ranges give lower limits of the bulk kinetic energy of the $\mathrm{H}_{2}$ gas for each positions. An integration over the full extent of the $\mathrm{CO}$ velocity range gives an upper limit (see Table 3).

The $\mathrm{CO}$ emitting gas carries a huge amount of kinetic energy, $\approx 4 \times 10^{57} \mathrm{erg}$, when summing on all positions. The kinetic energy of the $\mathrm{H}_{2}$ gas is at least five times higher than the thermal energy of the hot plasma heated by the galaxy collision (Guillard et al. 2009, 2010; estimated that this thermal energy is $\approx 9 \times 10^{56} \mathrm{erg}$ for the entire ridge and bridge). Before the collision, much of the kinetic energy of the gas is associated with the motions of the intruder and intra-group H I gas. The pre-shock H I mass in the area covered by the CO observations is estimated to be $1.0-2.8 \times 10^{9} M_{\odot}$ (based on an extrapolation of the Hi observations in NGC 7319's tidal tail to the area of the shock; see Section 2.2.1 of Guillard et al. 2009). Assuming that this gas is shocked at a velocity of $600 \mathrm{~km} \mathrm{~s}^{-1}$, the preshock H I kinetic energy is $0.5-1.3 \times 10^{58} \mathrm{erg}$. Therefore, the $\mathrm{CO}$ emitting gas is carrying a large fraction of the pre-shock kinetic energy of the gas. Most of this kinetic energy has not been thermalized yet. We suggest that this turbulent kinetic energy is the energy reservoir that powers the $\mathrm{H}_{2}$ line emission and maintains a large fraction of the mass of the $\mathrm{H}_{2}$ gas warm in SQ.

\subsection{Turbulent Heating of the Molecular Gas}

To assess the physical state of the molecular gas, we first compute the ratio between the warm $\mathrm{H}_{2}$ mass derived from the Spitzer/IRS spectroscopy and the $\mathrm{H}_{2}$ mass derived from the $\mathrm{CO}(1-0)$ observations, which we call "mid-IR/CO $\mathrm{H}_{2}$ mass ratio." These ratios are listed for each observed position in Table 2. On average, this ratio is much higher in SQ than in star-forming galaxies, where the mass of cold molecular gas $(<100 \mathrm{~K}$, usually traced by $\mathrm{CO})$ is $25-250$ times greater than the warm $(>100 \mathrm{~K}) \mathrm{H}_{2}$ mass detected in the mid-IR (Roussel et al. 2007). The $\mathrm{H}_{2}$ mass in the ridge derived from the $\mathrm{CO}(1-0)$ line emission is only a factor of $\approx 5$ larger than the warm $\mathrm{H}_{2}$ mass seen in the mid-IR domain $\left(8.8 \times 10^{8} M_{\odot}\right.$; see Table 7$)$. Surprisingly, this $\mathrm{H}_{2}$ mass ratio is extreme in SQ-A, whereas in the southern star-forming region (ridge 3 ) it is the lowest, with a value $(0.1)$ compatible with star-forming galaxies.

The mid-IR/CO $\mathrm{H}_{2}$ mass ratios in SQ are comparable to those observed in molecular clouds near the Galactic center (Rodríguez-Fernández et al. 2001), or in the bridge between the Taffy galaxies (UGC $12914 / 5$ ), where $0.1 \lesssim M_{\mathrm{H}_{2}}^{(\mathrm{w})} / M_{\mathrm{H}_{2}}^{(\mathrm{c})} \lesssim 0.7$ (B. W. Peterson et al., submitted), or in $\mathrm{H}_{2}$-luminous radio galaxies (Ogle et al. 2010). In all these environments, it has been concluded that the molecular gas is heated by shocks rather than UV photons. In such environments, the $\mathrm{CO}$ emission may trace not only cold molecular gas $(\approx 10-100 \mathrm{~K})$ but also warmer gas $\left(\approx 100\right.$ to a few $10^{3} \mathrm{~K}$, like the gas traced by mid-IR rotational $\mathrm{H}_{2}$ lines).

The $\mathrm{H}_{2}$ luminosity-to-mass ratio, $\mathcal{L}\left(\mathrm{H}_{2}\right) / \mathrm{M}\left(\mathrm{H}_{2}\right)$, measures the cooling rate (per unit mass) of the molecular gas (Guillard et al. 2009; Nesvadba et al. 2010). We compare this ratio to the turbulent heating rate per unit mass, written as $\Gamma_{\mathrm{T}}=$ $(3 / 2) \sigma_{T}^{2} / t_{\text {dis }}$ (Mac Low 1999). $\sigma_{T}$ is the turbulent velocity dispersion of the $\mathrm{H}_{2}$ gas and $t_{\mathrm{dis}}$ is the dissipation timescale, often written as $t_{\text {dis }}=l / \sigma_{T}$, where $l$ is the size of the region over which the velocity dispersion is measured. If the $\mathrm{H}_{2}$ emission is powered by the dissipation of turbulent energy, then the total energy dissipation rate must be at least equal to the $\mathrm{H}_{2}$ luminosity (Guillard et al. 2009):

$$
\frac{3}{2} \frac{\sigma_{T}^{3}}{l} \geqslant \frac{\mathcal{L}\left(\mathrm{H}_{2}\right)}{M\left(\mathrm{H}_{2}\right)}
$$

On average in the SQ IGM, we find $\mathcal{L}\left(\mathrm{H}_{2}\right) / \mathrm{M}\left(\mathrm{H}_{2}\right)=0.10 \pm$ $0.02 L_{\odot} M_{\odot}^{-1}$. At the spatial scales of a giant molecular cloud (GMC; $l \approx 100 \mathrm{pc}$ ), this condition implies $\sigma_{T}>35 \mathrm{~km} \mathrm{~s}^{-1}$. This is an order of magnitude higher than the $\mathrm{CO}$ velocity dispersion observed in Galactic GMCs at these spatial scales (Heyer et al. 2009), which shows that the molecular gas in SQ is much more turbulent than in the Galaxy. 


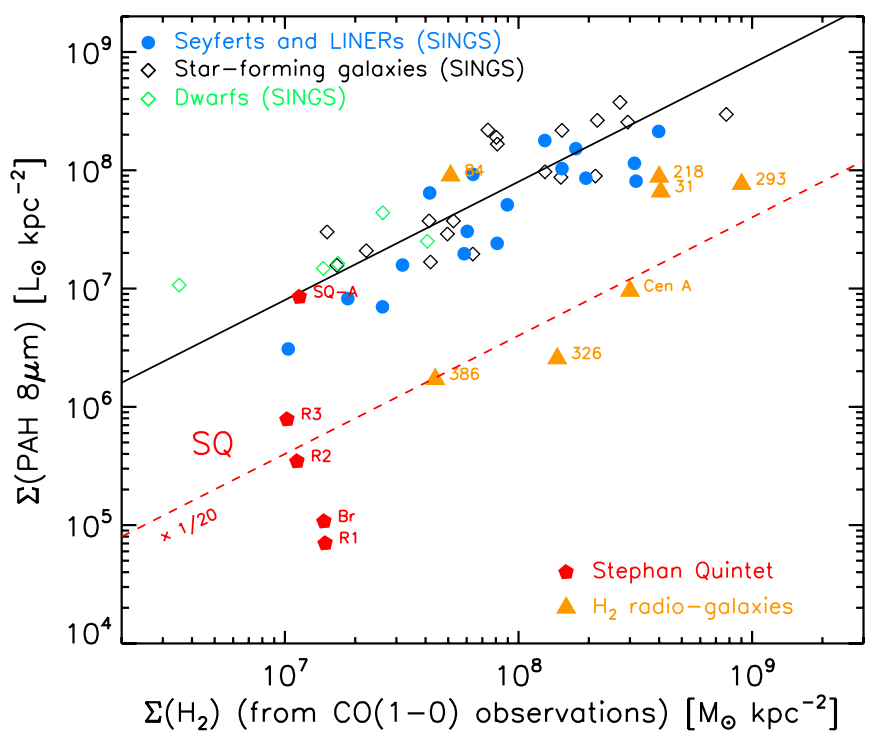

Figure 5. Star formation rate (assumed to be traced by the surface luminosity of the PAH emission) as a function of the surface mass of $\mathrm{H}_{2}$, derived from $\mathrm{CO}(1-0)$ line measurements, integrated over the full velocity range. The solid line shows the relationship obtained for the SINGS sample. Empty diamonds and filled dots mark dwarf galaxies, star-forming galaxies, and AGNs. The SINGS data are from Roussel et al. (2007). The filled red pentagons show the positions within the Stephan's Quintet group where we performed CO observations and extracted IRS spectra. The R1, R2, and R3 are the three positions in the ridge. $\mathrm{SQ}-\mathrm{A}$ denotes the northern star-forming region and $\mathrm{Br}$ denotes the bridge (see Figure 1). For comparison, the orange triangles mark the $\mathrm{H}_{2}$-luminous radio galaxies of the Ogle et al. (2010) sample for which we have both clear PAH and $\mathrm{CO}$ detections.

(A color version of this figure is available in the online journal.)

\section{DISCUSSION}

These $\mathrm{CO}$ observations raise questions related to the efficiency of star formation in the IGM and the origin of the $\mathrm{CO}$ gas.

\subsection{Shocks, Star Formation, and Physical State of the Molecular Gas in the SQ Group}

Following Nesvadba et al. (2010), Figure 5 presents a diagram similar to the classical Schmidt-Kennicutt relationship. Based on the empirical correlation between polycyclic aromatic hydrocarbon (PAH) emission and star formation in starbursts (Calzetti et al. 2007; Pope et al. 2008), the $7.7 \mu \mathrm{m}$ PAH emission is used as a tracer of star formation. The intensities of the PAH complexes measured on the IRS spectra are listed in Table 8.

We find that the SQ shock and bridge regions are significantly offset (up to -1.9 dex) from the correlation obtained with the SINGs sources (data taken from Roussel et al. 2007). Similar low PAH-to-CO surface luminosity ratios are also found in some $\mathrm{H}_{2}$-luminous radio galaxies (Nesvadba et al. 2010). We interpret this relative suppression of PAH emission as evidence for a lower star formation efficiency.

Part of this offset could come from an underabundance of PAHs with respect to the Galactic value (possibly caused by their destruction in shocks). Our modeling of the dust emission showed that the observed PAH and mid-IR continuum emission are consistent with the expected emission from the warm $\mathrm{H}_{2}$ gas in the shock for a Galactic dust-to-gas mass ratio and Galactic PAHs and very small grains abundances (Guillard et al. 2010). Since the $\mathrm{H}_{2}$ mass derived from the $\mathrm{CO}$ observations is only a factor of five (or less, given the uncertainties on the $X$-factor)
Table 8

Intensities of the PAHs Complexes

\begin{tabular}{lcccc}
\hline \hline Target Region & $7.7 \mu \mathrm{m}$ & $11.3 \mu \mathrm{m}$ & $12.6 \mu \mathrm{m}$ & $17 \mu \mathrm{m}$ \\
\hline SQ-A & & & & $4.67 \pm 0.21$ \\
Ridge 1 & $1.54 \pm 1.20$ & $8.53 \pm 0.48$ & $2.23 \pm 0.22$ & $1.84 \pm 0.36$ \\
Ridge 2 & $7.60 \pm 1.09$ & $7.59 \pm 0.26$ & $3.01 \pm 0.26$ & $2.79 \pm 0.32$ \\
Ridge 3 & $17.2 \pm 1.44$ & $9.31 \pm 0.27$ & $1.80 \pm 0.37$ & $1.50 \pm 0.27$ \\
Bridge & & & & $0.96 \pm 0.26$ \\
\hline
\end{tabular}

Notes. PAH intensities derived from Spitzer IRS spectra at the positions of our $\mathrm{CO}$ observations and integrated over the $\mathrm{CO}(1-0)$ beam. Fluxes are in units of $10^{-17} \mathrm{~W} \mathrm{~m}^{-2}$.

higher than the $\mathrm{H}_{2}$ mass derived from IRS spectroscopy, it is unlikely that the PAH abundance would be depleted by more than an order of magnitude. Therefore, we favor the possibility that the efficiency of conversion from molecular gas to stars in the shock is much lower than in "normal" star-forming galaxies. On the contrary, the SQ-A region is consistent with these galaxies and with star-forming collision debris in colliding galaxies (Boquien et al. 2010). In this region, the star formation rate is more than one order of magnitude higher than in the shock (Cluver et al. 2010).

Interestingly, we find that the regions where the star formation is the most suppressed are those where the $\mathrm{CO}$ velocity dispersion is the highest. The very high velocity dispersion observed in the CO gas in the SQ shock and bridge could maintain most of this gas in a diffuse and non-gravitationally bound state, which would explain why this molecular gas is inefficient at forming stars. This trend is also observed in other interacting systems like the TDG VCC 2062 (Duc et al. 2007) or Arp 94 (Lisenfeld et al. 2008), where the star formation is only observed in regions where the CO lines have a small velocity dispersion. Suppression of star formation in highly turbulent molecular disks has also been observed in radio galaxies (Nesvadba et al. 2011).

\subsection{Origin of the CO Gas in the IGM of $S Q$ : Pre- or Post-shock Gas?}

The CO gas in the IGM of SQ has two possible origins: it is either associated with the pre-shock or the post-shock gas (see the discussion in Guillard et al. 2009).

We call pre-shock molecular gas the $\mathrm{H}_{2}$ clouds that have not seen the fast $\left(\approx 600 \mathrm{~km} \mathrm{~s}^{-1}\right)$ intercloud shock produced by the galaxy collision (materialized by the X-ray emitting ridge), or the clouds for which the fast intercloud shock have swept across them, but these clouds have survived to the shock. In the latter case, these pre-shock molecular clouds must be dense enough not to be ionized by the radiation field of the fast intercloud shock (propagating in the tenuous, $n_{\mathrm{H}} \approx$ $10^{-2} \mathrm{~cm}^{-3}$, and X-ray emitting gas). Such clouds have too much inertia to be decelerated by the tenuous gas and retain their preshock velocity (in the Appendix we show that the deceleration timescale is longer than the age of the shock). Therefore, we expect to detect this $\mathrm{CO}$ gas at velocities close to the two preshock velocities (5700-6000 and $6700 \mathrm{~km} \mathrm{~s}^{-1}$ ).

We call post-shock gas the molecular gas that is formed from diffuse gas (e.g., H I gas) that has been heated by the transmitted shocks into the clouds, decelerated in the frame of reference of the intercloud shock front, and that had time to cool and become molecular within this deceleration timescale. This is the scenario that Guillard et al. (2009) proposed to explain the formation of $\mathrm{H}_{2}$ in the SQ ridge. This post-shock $\mathrm{H}_{2}$ gas is dynamically coupled to the lower density phases. In the frame 
Table 9

Mass, Energy, and Luminosity Budgets of the Pre-shock and Post-shock Gas in the SQ Ridge

\begin{tabular}{|c|c|c|c|c|c|c|c|}
\hline \multirow[b]{2}{*}{ Gas Phases } & \multicolumn{3}{|c|}{ Pre-shock Gas } & \multicolumn{4}{|c|}{ Post-shock Gas } \\
\hline & Hot Plasma ${ }^{a}$ & $\mathrm{HI}^{\mathrm{b}}$ & $\mathrm{H}_{2}{ }^{\mathrm{e}}$ & Hot Plasma ${ }^{a}$ & $\mathrm{H} \mathrm{II}^{\mathrm{c}}$ & $\mathrm{HI}^{\mathrm{b}}$ & $\mathrm{H}_{2}{ }^{\mathrm{e}}$ \\
\hline$n_{\mathrm{H}}\left(\mathrm{cm}^{-3}\right)$ & $3.2 \times 10^{-3}$ & $\cdots$ & $\ldots$ & $1.17 \times 10^{-2}$ & $\cdots$ & $\cdots$ & $\cdots$ \\
\hline$T(\mathrm{~K})$ & $5.7 \times 10^{6}$ & $\cdots$ & $10-10^{3}$ & $6.9 \times 10^{6}$ & $\cdots$ & $\cdots$ & $10-10^{3}$ \\
\hline$P / k_{\mathrm{B}}\left(\mathrm{K} \mathrm{cm}^{-3}\right)$ & $\ldots$ & $\ldots$ & $\ldots$ & $1.9 \times 10^{5}$ & $\ldots$ & $\ldots$ & $\ldots$ \\
\hline$N_{\mathrm{H}}\left(\mathrm{cm}^{-2}\right)$ & $\cdots$ & $3 \times 10^{20}$ & $\cdots$ & $\ldots$ & $1.4 \times 10^{19}$ & $<5.8 \times 10^{19}$ & $5 \times 10^{20}$ \\
\hline Masses $\left(M_{\odot}\right)$ & $2.6 \times 10^{8}$ & $1.0-2.8 \times 10^{9}$ & $<2.5 \times 10^{9}$ & $5.3 \times 10^{8}$ & $4.9 \times 10^{7}$ & $<2 \times 10^{8}$ & $1.6-4.1 \times 10^{9}$ \\
\hline \multirow[t]{2}{*}{ Energy (erg) } & Thermal & \multicolumn{2}{|c|}{ Kinetic } & Thermal & & & Kinetic \\
\hline & $3.4 \times 10^{56}$ & $0.5-1.3 \times 10^{58}$ & $<1.9 \times 10^{57}$ & $9 \times 10^{56}$ & $6 \times 10^{55}$ & $\cdots$ & $4-13 \times 10^{57}$ \\
\hline Flux $\left(\mathrm{W} \mathrm{m}^{-2}\right)$ & $\begin{array}{c}\text { X-rays } \\
6.6 \times 10^{-17} \\
\end{array}$ & $\cdots$ & $\begin{array}{c}\mathrm{CO}(1-0) \\
<1.9 \times 10^{-17} \\
\end{array}$ & $\begin{array}{c}\text { X-rays } \\
31.4 \times 10^{-17}\end{array}$ & $\begin{array}{c}\mathrm{H} \alpha \\
7.8 \times 10^{-17}\end{array}$ & $\begin{array}{c}\text { O I } \\
5.5 \times 10^{-17} \\
\end{array}$ & $\begin{array}{c}{\text { mid-IR } \mathrm{H}_{2} \text { lines }}^{\mathrm{d}} \\
7.5 \times 10^{-16}\end{array}$ \\
\hline $\mathcal{L}^{\mathrm{f}}\left(\operatorname{erg~s}^{-1}\right)$ & $6.2 \times 10^{40}$ & $\cdots$ & $<2 \times 10^{40}$ & $2.95 \times 10^{41}$ & $6.5 \times 10^{40}$ & $4.6 \times 10^{40}$ & $8 \times 10^{41}$ \\
\hline
\end{tabular}

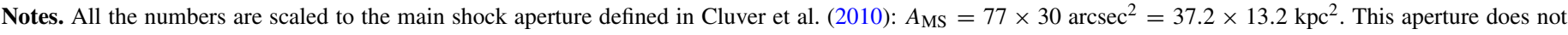

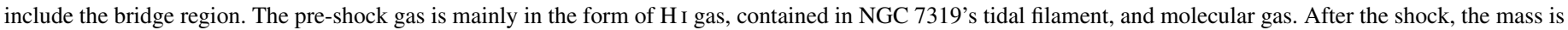
distributed between the hot X-ray emitting plasma and the $\mathrm{H}_{2}$ gas (see the discussion in Guillard et al. 2009).

a Chandra observations of the extended X-ray emission in the shock and the tidal tail (O'Sullivan et al. 2009a).

${ }^{b}$ Based on extrapolation of H I observations in the tidal tail by Sulentic et al. (2001) and Williams et al. (2002).

${ }^{\mathrm{c}} \mathrm{H} \alpha$ and O I optical line observations by Xu et al. (2003).

${ }^{\mathrm{d}}$ From Spitzer IRS observations. The $\mathrm{H}_{2}$ line flux is summed over the $\mathrm{S}(0)$ to $\mathrm{S}(5)$ lines (Cluver et al. 2010).

e Derived from the $\mathrm{CO}$ observations presented in this paper, assuming a Galactic conversion factor.

${ }^{\mathrm{f}}$ Luminosities are indicated assuming a distance to SQ of $94 \mathrm{Mpc}$.

of reference of the observer, the post-shock $\mathrm{CO}$ gas, formed from shocked intruder gas, is decelerated to velocities redder than the pre-shock intruder velocity (at $V \gtrsim 5700-6000 \mathrm{~km} \mathrm{~s}^{-1}$ ), while the post-shock CO gas, formed from shocked IGM gas, ${ }^{15}$ is decelerated to velocities bluer than the pre-shock IGM velocity $\left(V \lesssim 6700 \mathrm{~km} \mathrm{~s}^{-1}\right)$. Therefore, some post-shock CO gas should appear at a velocity intermediate to that of the two colliding gas flows. However, depending on the coupling efficiency and the dynamics of the galaxies in the group, some post-shock gas could also be found close to the IGM (NGC 7319's tidal debris) or the intruder (NGC 7318b) velocities.

The detection of $\mathrm{CO}$ emitting gas at both pre-shock and intermediate velocities between that of the intruder and that of the IGM shows that the molecular gas in the IGM of SQ originates from both pre-shock clouds, and from the formation of molecular gas in the cooling post-shock gas that has been accelerated in the frame of the shock. The observed CO kinematics show that the pre-shock components have lower velocity dispersions than the post-shock components (Section 4.3), suggesting that the shock is driving turbulence into the post-shock gas.

\subsection{Pre- and Post-shock Gas Masses and Energy Budgets}

The mass and energy budget of the pre-shock and postshock gas in the ridge is summarized in Table 9, which is an updated version of Table 1 in Guillard et al. (2009), now taking into account the $\mathrm{CO}$ data and the $\mathrm{H}_{2}$ spectral mapping of the shock (ridge) region by Cluver et al. (2010). Distinguishing spatially between the pre- and post-shock gas is difficult with the present $\mathrm{CO}$ observations. We rather rely on the kinematics to separate and quantify the masses and kinetic energies carried by each component. The uncertainties in the relative masses associated with both components are high, essentially because the dynamical coupling of the $\mathrm{H}_{2}$ gas with lower density phases and the global dynamics of the system are poorly constrained.

\footnotetext{
${ }^{15} \mathrm{CO}$ should form on a similar timescale as $\mathrm{H}_{2}$ in the post-shock gas (see
} Figure C.2 of Guillard et al. 2009).
In Table 9, the $\mathrm{CO}$ components with central velocities in the ranges $5700-6100$ and $6600-6800 \mathrm{~km} \mathrm{~s}^{-1}$ are associated with the pre-shock gas of the intruder and the intra-group medium, according to $\mathrm{HI}$ (Sulentic et al. 2001) and optical data (Xu et al. 2003). Outside these ranges, the CO emission is counted as post-shock gas. These velocity boundaries are highly uncertain and are likely to underestimate the proportion of post-shock gas and overestimate the pre-shock gas masses. We thus consider the post-shock gas masses as lower limits and pre-shock gas masses as upper limits. In particular we choose to include the $6000 \mathrm{~km} \mathrm{~s}^{-1}$ component in the pre-shock budget as suggested in Moles et al. (1997), Sulentic et al. (2001), and $\mathrm{Xu}$ et al. (2003), but this is questioned by other authors (Williams et al. 2002; Lisenfeld et al. 2002) who argue this is intruder gas that has been accelerated by the shock from 5700 to $6000 \mathrm{~km} \mathrm{~s}^{-1}$.

Under this assumption, the post-shock cold $\mathrm{H}_{2}$ mass is $\approx 1 / 3$ of the mass detected at the pre-shock velocities. Alternatively, if we consider that the $6000 \mathrm{~km} \mathrm{~s}^{-1}$ component belongs to the post-shock gas, this proportion is $\approx 3 / 2$. In either case, both pre-shock origin and post-shock $\mathrm{CO}$ formation are important.

The kinetic energy associated with pre- or post-shock gas has been calculated from Equation (3), based on the same velocity separation as for the masses (Section 7.3). For the post-shock gas, we quote the range of kinetic energy, being computed velocity component by component, or integrated over the full velocity range (after subtraction of the $\mathrm{CO}$ signal at the preshock velocities).

\section{CONCLUSIONS AND FINAL REMARKS}

We report on $\mathrm{CO}(1-0),(2-1)$, and (3-2) line observations with the IRAM $30 \mathrm{~m}$ and APEX telescopes in the galaxy-wide shock of $\mathrm{SQ}$, aimed at studying the $\mathrm{CO}$ gas counterpart to the powerful warm $\mathrm{H}_{2}$ emission detected by Spitzer/IRS. The main results are the following. 
1. Multiple CO velocity components are detected, at the pre-shock velocities (namely, that of the intruder galaxy at $5700 \mathrm{~km} \mathrm{~s}^{-1}$ and that of the intra-group medium at $\left.6700 \mathrm{~km} \mathrm{~s}^{-1}\right)$, but also at intermediate $\left(6000-6400 \mathrm{~km} \mathrm{~s}^{-1}\right)$ and higher (6900 $\mathrm{km} \mathrm{s}^{-1}$ in SQ-A) velocities. The CO gas originates from both pre-shock molecular gas and postshock gas, i.e., diffuse gas heated by the shock that had time to cool and become molecular within the collision age. $\mathrm{CO}$ lines are intrinsically broader in the ridge and in the bridge than in the SQ-A and southern star-forming regions. A large fraction of the gas kinetic energy of the collision is carried by the turbulent velocity dispersion of the $\mathrm{CO}$ emitting gas, which means that most of this energy has not been thermalized yet.

2. The $\mathrm{CO}(1-0)$ and (2-1) emission is distributed over the intra-group shock (the ridge) and in an eastern bridge feature that connects the shock to NGC 7319. We also detected the $\mathrm{CO}(3-2)$ line in the ridge and tentatively in SQ-A. Large amounts of CO emitting gas are detected, $\approx 5.4 \times 10^{9} M_{\odot}$ (after conversion from $\mathrm{CO}(1-0)$ emission to $\mathrm{H}_{2}$ using the Galactic value), in the ridge and in the bridge. Note that this mass could be lower by a factor of a few because of the large uncertainties on the $\mathrm{CO}$ to $\mathrm{H}_{2}$ conversion factor. This gas is cospatial with warm $\mathrm{H}_{2}$ seen in the mid-IR domain, H II gas, and X-ray emitting hot plasma. The ratio between the $\mathrm{H}_{2}$ mass derived from the Spitzer spectroscopy to the $\mathrm{H}_{2}$ mass derived from the $\mathrm{CO}$ observations is $\approx 0.2$, which is $1-2$ orders of magnitude higher than in star-forming galaxies, showing that the heating rate of the molecular gas in SQ is particularly high. This high heating rate is likely to be powered by the turbulent dissipation of the kinetic energy involved in the galaxy collision. This interpretation implies that the velocity dispersion on the scale of GMCs in SQ is an order of magnitude larger than the Galactic value.

3. We find that the ratio of the PAH-to-CO surface luminosities in the shock and in the bridge regions are much lower (up to a factor 75) than the observed values in star-forming galaxies. In the SQ-A star-forming region, the PAH-to-CO ratio is compatible with the classical Schmidt-Kennicutt law. We suggest that the star formation efficiency in the shock of SQ is lower than in "normal" galaxies. This could be a consequence of the high turbulence within the $\mathrm{H}_{2}$ gas.

Our census of the molecular gas in the IGM of SQ is still incomplete. High angular resolution interferometric radio observations of SQ will help to determine the clumpiness of the $\mathrm{CO}$ emitting gas and perhaps spatially separate the different velocity components. Far-infrared photometry is also needed to estimate the total dust mass, and thus the cold gas content independently of the $\mathrm{CO}$ to $\mathrm{H}_{2}$ conversion factor.

A global model for the formation and kinematics of the molecular gas present in the IGM of SQ can only be addressed by means of complex numerical simulations. A first step has been achieved by Renaud et al. (2010), who presented a pilot $N$-body study with a solution that matches the overall geometry of the group. Hydrodynamical simulations by Hwang et al. (2012) took a step further including the gas component and made first headways in our understanding of the gas kinematics in the system. However, presently, the existing numerical simulations do not include the molecular gas and cannot reproduce the $\mathrm{CO}$ and $\mathrm{H}_{2}$ observations of SQ presented here and in Appleton et al. (2006) and Cluver et al. (2010), where most of the molecular gas is not associated with star formation. The prescriptions used in the numerical models to describe the conversion from interstellar gas to stars will have to be refined, especially in the treatment of the gas cooling, energy exchanges between the phases of the interstellar medium, and turbulent cascade of the kinetic energy of the collision.

This paper is based on observations carried out with the IRAM $30 \mathrm{~m}$ telescope. IRAM is supported by INSU/CNRS (France), MPG (Germany), and IGN (Spain). P.G. also would like to acknowledge in particular the IRAM staff for help provided during the observations. U.L. acknowledges support by the research project AYA2007-67625-C02-02 from the Spanish Ministerio de Ciencia y Educación and the Junta de Andalucía (Spain) Grant FQM-0108.

Part of this publication is based on data acquired with the Atacama Pathfinder EXperiment (APEX). APEX is a collaboration between the Max-Planck-Institut für Radioastronomie, the European Southern Observatory, and the Onsala Space Observatory.

This work is partly based on observations made with the Spitzer Space Telescope, which is operated by the Jet Propulsion Laboratory, California Institute of Technology, under a contract with NASA.

This research has made use of the NASA/IPAC Extragalactic Database (NED) which is operated by the Jet Propulsion Laboratory, California Institute of Technology, under contract with the National Aeronautics and Space Administration.

\section{APPENDIX \\ DYNAMICAL COUPLING OF THE CO GAS TO OTHER GAS PHASES}

In the following we show that it is unlikely that the $\mathrm{CO}$ gas observed at intermediate velocities originates from preshock molecular clouds, essentially because the timescale of dynamical coupling, i.e., the time it takes for the pre-shock cloud to decelerate and become comoving with the shocked intercloud medium, is much longer than the age of the collision.

Let us consider the case of an $\mathrm{H}_{2}$ cloud of density $\rho_{c}$ and radius $R_{c}$, initially in pressure equilibrium with the intercloud medium (of density $\rho_{i}$ ), that is run over by a fast shock wave of velocity $V_{i}$ in the intercloud medium. The timescale for the cloud compression due to the rise of the external pressure, socalled the crushing time, is given by $t_{\text {crush }}=R_{c} / V_{c}$, where $V_{c} \simeq \sqrt{\left(\rho_{i} / \rho_{c}\right)} V_{i}=V_{i} / \sqrt{\chi}$ is the velocity of the transmitted shock into the cloud $\left(\chi=\rho_{c} / \rho_{i}\right.$ is the density contrast between the two media). The cloud acceleration timescale, $t_{\mathrm{acc}}$, i.e., the amount of time it takes to accelerate the cloud to the velocity of the post-shock background $V_{\mathrm{ps}, \mathrm{i}}=(3 / 4) V_{i}$ is (e.g., Klein et al. 1994):

$$
\begin{gathered}
t_{\mathrm{acc}}=\frac{16}{9} \frac{\rho_{\mathrm{c}}}{\rho_{i}} \frac{R_{\mathrm{c}}}{V_{\mathrm{i}}}=\frac{16}{9} \sqrt{\chi} t_{\mathrm{crush}} \\
\simeq 1.7 \times 10^{8}\left(\frac{\chi}{10^{3}}\right)\left(\frac{R_{\mathrm{c}}}{10 \mathrm{pc}}\right)\left(\frac{V_{\mathrm{i}}}{100 \mathrm{~km} \mathrm{~s}^{-1}}\right)^{-1}(\mathrm{yr}) .
\end{gathered}
$$

The above equation shows that it takes a long time to couple dynamically pre-shock dense $\mathrm{H}_{2}$ gas with the background flow of tenuous gas. The cloud crushing time (time for the intercloud shock to go through the cloud) to acceleration time ratio is $t_{\text {crush }} / t_{\text {acc }}=9 / 16 \sqrt{\chi}$, which shows that as soon as the density 
contrast is higher than $\approx 10^{2}$, the cloud will be compressed on a timescale much shorter than the acceleration time. In the SQ shock, the high-density contrast between cold clouds and the hot tenuous flow ( $\chi>10^{5}$; see Guillard et al. 2009) would lead to deceleration timescales longer than the shock age.

On the other hand, in the model of $\mathrm{H}_{2}$ formation in the shock proposed by Guillard et al. (2009), the molecular gas is formed out of moderate density medium $\left(n_{\mathrm{H}} \approx 1 \mathrm{~cm}^{-3}\right)$ that is compressed and decelerated while becoming molecular. Therefore, in this context, the coupling is much more efficient because the density contrast is much lower $\left(\chi<10^{2}\right)$. This in situ formation is a solution to explain the high-velocity dispersion seen in the spectra, although we do not exclude the presence of pre-shock dense clouds.

\section{REFERENCES}

Aoki, K., Ohtani, H., Yoshida, M., \& Kosugi, G. 1996, AJ, 111, 140 Appleton, P. N., Xu, K. C., Reach, W., et al. 2006, ApJ, 639, L51 Arp, H. 1973, ApJ, 185, 797

Boquien, M., Bendo, G., Calzetti, D., et al. 2010, ApJ, 713, 626

Boquien, M., Duc, P.-A., Wu, Y., et al. 2009, AJ, 137, 4561

Braine, J., Davoust, E., Zhu, M., et al. 2003, A\&A, 408, L13

Braine, J., Duc, P.-A., Lisenfeld, U., et al. 2001, A\&A, 378, 51

Calzetti, D., Kennicutt, R. C., Engelbracht, C. W., et al. 2007, ApJ, 666, 870

Cluver, M. E., Appleton, P. N., Boulanger, F., et al. 2010, ApJ, 710, 248

Crutcher, R. M. 1999, ApJ, 520, 706

Downes, D., \& Solomon, P. M. 1998, ApJ, 507, 615

Duc, P., Braine, J., Lisenfeld, U., Brinks, E., \& Boquien, M. 2007, A\&A, 475, 187

Flower, D. R., \& Pineau des Forêts, G. 2010, MNRAS, 406, 1745

Gao, Y., \& Xu, C. 2000, ApJ, 542, L83

Gao, Y., Zhu, M., \& Seaquist, E. R. 2003, AJ, 126, 2171

Guillard, P. 2010, PhD thesis, IAS, Université Paris Sud 11, arXiv:1001.3613

Guillard, P., Boulanger, F., Cluver, M. E., et al. 2010, A\&A, 518, A59

Guillard, P., Boulanger, F., Pineau Des Forêts, G., \& Appleton, P. N. 2009, A\&A, 502,515

Güsten, R., Nyman, L. Å., Schilke, P., et al. 2006, A\&A, 454, L13

Harwit, M., Houck, J. R., Soifer, B. T., \& Palumbo, G. G. C. 1987, ApJ, 315, 28

Heyer, M., Krawczyk, C., Duval, J., \& Jackson, J. M. 2009, ApJ, 699, 1092

Hwang, J.-S., Struck, C., Renaud, F., \& Appleton, P. 2012, MNRAS, 419, 1780

Iglesias-Páramo, J., López-Martín, L., Vílchez, J. M., Petropoulou, V., \& Sulentic, J. W. 2012, arXiv:1201.4030

Joseph, R. D., \& Wright, G. S. 1985, MNRAS, 214, 87

Kim, D., Sanders, D. B., Veilleux, S., Mazzarella, J. M., \& Soifer, B. T. 1995, ApJS, 98, 129
Klein, R. I., McKee, C. F., \& Colella, P. 1994, ApJ, 420, 213

Lee, H. M., Kang, H., \& Ryu, D. 1996, ApJ, 464, 131

Leon, S., Combes, F., \& Menon, T. K. 1998, A\&A, 330, 37

Lisenfeld, U., Braine, J., Duc, P.-A., et al. 2002, A\&A, 394, 823

Lisenfeld, U., Braine, J., Duc, P.-A., et al. 2004, A\&A, 426, 471

Lisenfeld, U., Mundell, C. G., Schinnerer, E., Appleton, P. N., \& Allsopp, J. 2008, ApJ, 685, 181

Mac Low, M.-M. 1999, ApJ, 524, 169

Maier, D., Barbier, A., Lazareff, B., \& Schuster, K. F. 2005, in Proc. 16th International Symposium on Space Terahertz Technology, ed. M. Ingrarson, J. Stake, \& H. Merkel (Göteborg: Chalmers Univ.), 428

Markwardt, C. B. 2009, in ASP Conf. Ser. 411, Astronomical Data Analysis Software and Systems XVIII, ed. D. A. Bohlender, D. Durand, \& P. Dowler (San Francisco, CA: ASP), 251

Moles, M., Sulentic, J. W., \& Marquez, I. 1997, ApJ, 485, L69

Natale, G., Tuffs, R. J., Xu, C. K., et al. 2010, ApJ, 725, 955

Nesvadba, N. P. H., Boulanger, F., Lehnert, M. D., Guillard, P., \& Salome, P. 2011, A\&A, 536, L5

Nesvadba, N. P. H., Boulanger, F., Salomé, P., et al. 2010, A\&A, 521, A65

Ogle, P., Boulanger, F., Guillard, P., et al. 2010, ApJ, 724, 1193

O’Sullivan, E., Giacintucci, S., Vrtilek, J. M., Raychaudhury, S., \& David, L. P. 2009a, ApJ, 701, 1560

O'Sullivan, E., Giacintucci, S., Vrtilek, J. M., et al. 2009b, in ASP Conference Series, 407, The Low-Frequency Radio Universe, ed. D. J. Saikia et al. (San Francisco, CA: ASP), 250

Petitpas, G. R., \& Taylor, C. L. 2005, ApJ, 633, 138

Pope, E. C. D., Hartquist, T. W., \& Pittard, J. M. 2008, MNRAS, 389, 1259

Renaud, F., Appleton, P. N., \& Xu, C. K. 2010, ApJ, 724, 80

Rodríguez-Fernández, N. J., Martín-Pintado, J., Fuente, A., et al. 2001, A\&A, 365,174

Roussel, H., Helou, G., Hollenbach, D. J., et al. 2007, ApJ, 669, 959

Smith, B. J., \& Struck, C. 2001, AJ, 121, 710

Smith, J. D. T., Armus, L., Dale, D. A., et al. 2007a, PASP, 119, 1133

Smith, J. D. T., Draine, B. T., Dale, D. A., et al. 2007b, ApJ, 656, 770

Sulentic, J. W., Rosado, M., Dultzin-Hacyan, D., et al. 2001, AJ, 122, 2993

Trinchieri, G., Sulentic, J., Breitschwerdt, D., \& Pietsch, W. 2003, A\&A, 401, 173

Trinchieri, G., Sulentic, J., Pietsch, W., \& Breitschwerdt, D. 2005, A\&A, 444, 697

Verdes-Montenegro, L., Yun, M. S., Perea, J., del Olmo, A., \& Ho, P. T. P. 1998, ApJ, 497, 89

Williams, B. A., Yun, M. S., \& Verdes-Montenegro, L. 2002, AJ, 123, 2417

Xu, C. K. 2006, in Publication of Purple Mountain Observatory (Lijiang, China), e-print arXiv:astro-ph/0601084

Xu, C. K., Iglesias-Páramo, J., Burgarella, D., et al. 2005, ApJ, 619, L95

Xu, C. K., Lu, N., Condon, J. J., Dopita, M., \& Tuffs, R. J. 2003, ApJ, 595, 665

Xu, C. K., Sulentic, J. W., \& Tuffs, R. 1999, ApJ, 512, 178

Yun, M. S., Verdes-Montenegro, L., del Olmo, A., \& Perea, J. 1997, ApJ, 475, L21 